\title{
Consumption and Portfolio Choice under Loss Aversion and Endogenous Updating of the Reference Level*
}

\author{
Servaas van Bilsen \\ Amsterdam School of Economics \\ University of Amsterdam \\ and NETSPAR
}

\author{
Roger J. A. Laeven ${ }^{\dagger}$ \\ Amsterdam School of Economics \\ University of Amsterdam \\ EURANDOM and CentER
}

\author{
Theo E. Nijman \\ Department of Finance \\ Tilburg University \\ CentER and NETSPAR
}

Thursday $28^{\text {th }}$ March, 2019

\begin{abstract}
We explicitly derive and explore the optimal consumption and portfolio policies of a lossaverse individual who endogenously updates his reference level over time. We find that he protects his current consumption by delaying painful reductions in consumption after a drop in wealth, and increasingly so with higher degrees of endogeneity. The incentive to protect current consumption is stronger with a medium wealth level than with a high or low wealth level. Furthermore, this individual adopts a conservative investment strategy in normal states and typically a more aggressive strategy in good and bad states. Endogeneity of the reference level increases overall risk-taking and generates an incentive to reduce risk exposure with age even without human capital. The welfare loss that this individual would suffer under the conventional CRRA consumption and portfolio policies easily exceeds $10 \%$.
\end{abstract}

JEL Classification: D81, D91, G02, G11.

OR/MS Classification: Risk, Investment, Portfolio.

Keywords: Loss Aversion; Endogenous Reference Level; Prospect Theory; Optimal Consumption Choice; Optimal Portfolio Choice.

\footnotetext{
${ }^{*}$ We are very grateful to the Department Editor, an Associate Editor, and two referees for comments and suggestions that have significantly improved the paper. We are also grateful to Yacine Aït-Sahalia, Lans Bovenberg, Nicole Branger, Conrad Ciccotello, Louis Eeckhoudt, Martin Grace, Michael Hoy, Gur Huberman, Frank de Jong, Olivia S. Mitchell, Andreas Richter, Nikolai Roussanov, Hans Schumacher, Kent Smetters, Sharon Tennyson, Jeremy Tobacman, Michel Vellekoop, Wei Xiong, and to conference and seminar participants at the Australasian Finance and Banking Conference, the Foundations of Utility and Risk Conference, the S.S. Huebner Foundation Colloquium, the Netspar International Pension Workshop, the Quantitative Methods in Finance Conference, CEAR at Georgia State University, East China Normal University Shanghai, the Center for Financial Studies at Goethe University Frankfurt, Tilburg University, the Tinbergen Institute, the University of Amsterdam, the University of Münster, and the University of Pennsylvania (Wharton School) for their helpful comments and suggestions. This research was supported in part by the Netherlands Organization for Scientific Research under grant NWO VIDI (van Bilsen, Laeven) and by the European Commission under grant EUMOPACT (van Bilsen, Nijman). Email addresses: S.vanBilsen@uva.nl; R. J.A.Laeven@uva.nl; Nyman@uvt.nl.

${ }^{\dagger}$ Corresponding author. Mailing Address: PO Box 15867, 1001 NJ Amsterdam, The Netherlands. Phone: $+31(0) 205254219$.
} 


\section{Introduction}

The conventional consumption and investment model dating back to Merton (1969) and based on an individual with CRRA (that is, iso-elastic marginal) utility suggests that the optimal drawdown ratio and optimal portfolio weight in the risky stock do not depend on past stock returns; only age and the individual's attitude towards risk and time play a role in determining the optimal strategies. This paper explores how loss aversion and reference dependence, which are two pronounced behavioral regularities that are supported by extensive experimental and empirical observations, jointly affect this conventional wisdom.

We show that the optimal policies of a loss-averse individual with a reference level that is endogenously determined by his own past consumption choices are fundamentally different from the conventional optimal policies 1 . His optimal drawdown ratio is not independent of the stock price, but typically rather increases as the stock price declines, and increasingly so if his reference level exhibits higher degrees of endogeneity. The extent to which a loss-averse individual adjusts his drawdown ratio in response to a stock price movement depends on his current wealth level: a medium-wealth individual has a stronger incentive to protect current consumption and increase the drawdown ratio after a drop in wealth than a low-wealth and, especially, a high-wealth individual.

The optimal portfolio weight of a loss-averse individual also differs from predictions of the conventional consumption and investment model. In particular, a loss-averse individual takes his reference level into account when making optimal portfolio decisions. As long as his reference level can just be financed, he implements a (very) conservative investment strategy, but if the financial market is in either boom or bust he typically increases stock holdings. Furthermore, endogeneity of the reference level yields a preference for adopting a life-cycle investment strategy that reduces risk exposure with age even without human capital, and increases overall risktaking. Hence, as we show in this paper, a loss-averse individual with an endogenous reference level who consumes and invests according to the conventional consumption and investment model suffers a substantial welfare loss.

Inspired by the seminal work of Kahneman and Tversky (1979) and Tversky and Kahneman (1992), we represent the individual's preferences over gains and losses in consumption, defined relative to an endogenous reference level, by the two-part power utility function inducing loss aversion. This utility function exhibits a kink at the reference level, so that losses hurt more than gains satisfy. Furthermore, we assume that the individual updates his reference level in each period based on his own past consumption experiences. As a result, a higher consumption level today leads to an increase in the reference level tomorrow.

We derive explicit closed-form solutions to the individual's consumption and investment

\footnotetext{
${ }^{1}$ Section 2 reviews the related literature, including papers with modern preference models, and discusses how our findings differ from these papers.
} 
problem. This is non-trivial. It is achieved by first invoking the solution technique developed in Schroder and Skiadas (2002). These authors propose a technique that enables us to convert the individual's optimization problem with endogenous updating of the reference level into an equivalent dual problem without endogenous updating of the reference level. Then, we solve the dual problem by using the martingale approach, and by using techniques proposed by Basak and Shapiro (2001) (see also Berkelaar, Kouwenberg, and Post (2004)) to deal with pseudo-concavity and non-differentiability aspects of the problem. We suitably adapt the latter techniques to our setting with intertemporal consumption. Finally, we transform the optimal solutions of the dual problem back into the optimal solutions of the individual's original problem.

We show that optimal current consumption depends on past stock returns. A loss-averse individual divides past stock returns into three categories: a normal past return in which current consumption remains at (or slightly above) the reference level; a good past return in which current consumption is substantially above the reference level; and a bad past return in which current consumption falls below the reference level. A loss-averse individual thus adopts a selfinsurance strategy to protect against downside risk. Only in genuinely adverse circumstances, he consumes below the reference level. In case of a normal past return, his optimal current drawdown ratio goes up as the stock price goes down. Furthermore, a loss-averse individual who endogenously updates his reference level strongly postpones reductions in current consumption following a drop in wealth. Indeed, with a decreasing endogenous reference level, reductions in future consumption levels affect utility less heavily than a reduction in his current consumption level. This strengthens the motive to delay painful reductions in consumption that, as we show, typically occurs already with a constant reference level. The incentive to postpone cuts in current consumption after a negative wealth shock is strongest in case of bad and normal past returns. In such circumstances, a loss-averse individual wants to either prevent that current consumption falls below the reference level (normal past returns) or limit the size of a loss (bad past returns). The flipside of this is that a loss-averse individual who endogenously updates his reference level does not fully translate a positive wealth shock into his current consumption level but saves precautionarily, and, as a result, exhibits an excessively smooth and excessively sensitive consumption path ${ }^{2}$

The extent to which current consumption is protected after a drop in wealth is found to be heterogeneous across wealth levels. If we assume a constant reference level, such protection is most pronounced for a medium-wealth individual, less pronounced for a low-wealth individual, and null for a high-wealth individual. At higher degrees of endogeneity of the reference level, protection of current consumption overall increases and the heterogeneity across wealth levels gradually diminishes.

\footnotetext{
${ }^{2}$ Consumption is excessively smooth if consumption under-responds to wealth shocks; consumption is excessively sensitive if past wealth shocks have predictive power for future consumption growth. Campbell and Deaton (1989) show that an excessively smooth consumption stream is also excessively sensitive.
} 
The optimal portfolio weight in the risky stock displays primarily a U-shaped pattern across states of the economy. In case of a normal past return, a loss-averse individual adopts a (very) conservative portfolio strategy to prevent that current consumption falls below the reference level. The optimal portfolio strategy is (much) less conservative in case of a good and a bad past return. Indeed, in case of a good past return, a relatively aggressive portfolio strategy most likely does not lead to a loss with respect to the reference level in the near future. In case of a bad past return, a relatively aggressive portfolio strategy will increase the chance of realizing a future gain with respect to the reference level, but can eventually deplete consumption.

Furthermore, we find that, unlike in the conventional consumption and investment model, a loss-averse individual with an endogenous reference level adopts a life-cycle investment strategy, which reduces risk exposure with age, even without taking human wealth into account 3 Intuitively, as the individual ages and the investment horizon shrinks, he has less time to absorb wealth shocks. As a result, to maintain a stable consumption pattern over the life-cycle, he reduces the exposure to the risky stock as he grows older. Finally, we find that endogeneity of the reference level induces the individual to adopt a less conservative portfolio strategy.

We also compute the welfare loss (in terms of the relative decline in certainty equivalent consumption) associated with the conventional CRRA strategy and analyze the roles played by the preference parameters. In the welfare analysis, the loss-averse individual delegates his drawdown and portfolio decisions to a professional asset manager. This asset manager only offers strategies that are based on CRRA utility. The loss-averse individual chooses the CRRA strategy that minimizes the difference between his optimal utility (i.e., the utility associated with the optimal loss-averse strategy) and his actual utility (i.e., the utility associated with the CRRA strategy). For realistic parameter values, we find that a loss-averse individual with a constant reference level suffers a minimum welfare loss of $3 \%$. If the individual has an endogenous reference level instead, the welfare loss is likely to exceed $10 \%$.

The remainder of this paper is structured as follows. Section 2 reviews the literature. Section 3 describes our consumption and investment model. Section 4 presents the optimal consumption and portfolio policies. Section 5 provides an extensive analysis of the optimal policies. Section 6 conducts the welfare analysis. Finally, we conclude in Section 7 .

\section{Literature}

The first to study the problem of optimal consumption and portfolio selection over an individual's life-cycle in a continuous-time setting was Merton (1969). 4 The Merton model

\footnotetext{
${ }^{3}$ A CRRA individual optimally invests a constant proportion of his total wealth in risky assets. Bodie, Merton, and Samuelson (1992) provide a rationale for a CRRA individual to implement a life-cycle investment strategy that reduces risk exposure with age based on human capital considerations; see also footnote 27.

${ }^{4}$ See also the seminal contributions by Mossin (1968) and Samuelson (1969).
} 
with CRRA utility suggests a consumption-to-total-wealth ratio and life-cycle investment strategy that depend on age and attitudes towards risk and time only. These optimal policies continue to serve as the dominant benchmark for drawdown and investment strategies deployed in practice. Merton's model has been generalized along many dimensions 5 We are the first to explicitly solve Merton's consumption-investment problem under prospect theory utility (Kahneman and Tversky (1979) and Tversky and Kahneman (1992)) and analyze its implications for both optimal intertemporal consumption and the corresponding optimal portfolio behavior in an integrated setting.

Researchers have successfully applied prospect theory in many other domains of economics. For instance, in the finance literature, elements of prospect theory have been used to explain aggregate stock market behavior (e.g., Benartzi and Thaler (1995)), asset prices and the cross section of stock returns (e.g., Shumway (1997), Barberis, Huang, and Santos (2001), Yogo (2008), Barberis and Huang (2008), Andries (2012) and Pagel (2017b)), and trading patterns (e.g., Shefrin and Statman (1985), Odean (1998), Barberis and Xiong (2009), and Meng and Weng (2017)). Only a handful of papers have explored the implications of prospect theory for either optimal consumption or portfolio behavior.

Importantly, Berkelaar et al. (2004) and Gomes (2005) were the first papers that examine the optimal portfolio choice of a loss-averse individual. Their settings differ from ours in a number of ways. First, while they define utility in terms of gains and losses in terminal wealth, we consider a setting where the individual draws utility from gains and losses in intertemporal consumption. Such a setting allows us to study how the optimal consumption and portfolio policies of a loss-averse individual change as time proceeds and risk resolves, which is our prime focus. Second, in our setting with intertemporal consumption, the reference level evolves endogenously over time, which is not the case in Berkelaar et al. (2004) and Gomes (2005). While Berkelaar et al. (2004) also find a U-shaped pattern for the optimal portfolio choice, we find that our loss-averse individual with an endogenous reference level pursues overall a riskier investment strategy and, furthermore, reduces his exposure to equity risk as age proceeds, even without considering human capital, contrary to in Berkelaar et al. (2004).

Jin and Zhou (2008) and He and Zhou (2011, 2016) explore the implications of loss aversion and, in particular, probability weighting for optimal portfolio holdings, developing a novel analytical solution technique based on a quantile formulation. These authors do not study intertemporal consumption choice, nor do they consider endogenous updating of the individual's reference level. Because probability weighting can lead to an even larger degree of protection against downside risk (see also, e.g., Carlson and Lazrak (2014)), we indicate in Section 5.5 how to include probability weighting into our setting with intertemporal consumption.

\footnotetext{
${ }^{5}$ These include, for example, generalizations to allow for risky labor income (e.g., Viceira (2001), Gomes and Michaelides (2005), Cocco, Gomes, and Maenhout (2005), and Benzoni, Collin-Dufresne, and Goldstein (2007)) and a stochastic investment opportunity set (e.g., Campbell, Cocco, Gomes, Maenhout, and Viceira (2001), Wachter (2002), Chacko and Viceira (2005), Liu (2007), and Laeven and Stadje (2014)).
} 
Prospect theory has also been incorporated into (personal) equilibrium models of optimal consumption behavior. The focus of these models is on the optimal consumption dynamics and not on the associated optimal portfolio behavior. A main example is Köszegi and Rabin (2009) who consider a discrete-time consumption and savings model in which the individual exhibits so-called expectations-based loss aversion. In Köszegi and Rabin's model, the individual draws utility from current consumption, and from changes in expectations about present and future consumption. The individual is loss-averse in the sense that losses in expectations hurt more than gains in expectations feel good. Furthermore, immediate losses affect utility more heavily than future losses. Although Köszegi and Rabin (2009) consider a different setting in which the updating rule for the reference level is forward-looking rather than (as in our case) backwardlooking and time is discrete, some of our model's implications for optimal consumption behavior are consistent with theirs. In particular, as shown by Pagel (2017a), Köszegi and Rabin's model also generates a (first-order) precautionary savings motive and an excessively smooth and excessively sensitive consumption stream. This is reassuring for the robustness of these implications with respect to the specification of the updating rule.

A backward-looking updating rule for the reference level has also been extensively used in the habit formation literature since Constantinides (1990). In this literature, the reference level serves as a minimum subsistence level rather than as a standard-of-living as in our general setting, and thus this literature obtains different results. The optimal portfolio weight in the risky stock for our individual is primarily $U$-shaped across states of the economy, while under the difference internal habit model it is increasing in the state of the economy. In particular, we show that the optimal portfolio strategy of a loss-averse individual with an endogenous reference level is typically more conservative than the optimal portfolio strategy of an individual with preferences that comply with the difference internal habit model.

Guasoni, Huberman, and Ren (2015) analyze the implications of 'shortfall aversion' for optimal consumption and portfolio behavior. These authors consider a ratio habit model (see Abel (1990) ) in which the reference level is given by past peak consumption, which yields a setting that is quite different from ours. Consistent with our results, they find that in normal states of the economy, consumption is constant at the reference level. However, while we find a U-shaped pattern for the optimal portfolio weight, they find that the optimal portfolio weight is an increasing function of wealth.

Recently, Curatola (2015, 2017) also explores a preference model that combines loss aversion with an endogenous reference level. While Curatola (2015) examines the asset-pricing implications, Curatola (2017) analyzes the implications for optimal consumption and portfolio choice. In Curatola (2017), the individual is restricted to be risk loving in the loss domain. Furthermore, he assumes that the reference level is a linear function of the difference between current consumption and an initial consumption level $]^{6}$ By contrast, we also allow for

\footnotetext{
${ }^{6}$ The habit level specification assumed in Curatola $(2015)$ differs from the standard habit formation literature,
} 
risk-averse behavior in the loss domain, and, even more importantly, represent the reference level by a weighted sum of the individual's past consumption choices, i.e., of his realized past consumption path. As a result of this substantial difference in model specification, our solution strategy differs methodologically considerably from the solution strategy in Curatola (2017). Furthermore, the focus of Curatola (2017) is different: he mainly studies the performance of optimal portfolios, while we analyze how optimal consumption and portfolio behavior varies across different individuals and how large the welfare costs are associated with loss aversion and endogenous updating.

Prospect theory is, of course, not the only behavioral economic theory that has been developed in the literature. Other behavioral theories include regret theory (Loomes and Sugden (1982), Bell (1982, 1983), Sugden (1993), and Quiggin (1994)) and disappointment (aversion) theory (Bell (1985), Loomes and Sugden (1986), and Gul (1991)). A number of authors have studied these theories in the context of (only) optimal portfolio selection. For instance, Muermann, Mitchell, and Volkman (2006) explore the optimal portfolio allocation problem of a regret-averse individual. They show that if the equity risk premium is high enough, a regret-averse individual invests less in the risky stock than a risk-averse individual. Intuitively, adopting an aggressive portfolio strategy strongly exposes a regret-averse individual to the likelihood of significant regret if stocks do poorly. A loss-averse individual also (typically) invests less in the risky stock than a risk-averse individual, but for a different reason. Indeed, a loss-averse individual aims to avoid losses rather than to prevent regret. Ang, Bekaert, and Lui (2005) explore the portfolio allocation decision of a disappointment-averse individual. Their model generates empirically reasonable equity portfolio allocations. In particular, the authors are able to explain the stock market participation puzzle (Mankiw and Zeldes (1991)).

\section{Model}

\subsection{Preferences}

Time is continuous. We denote by $t$ adult age, which corresponds to effective age minus 25 . To isolate the effects of loss aversion and an endogenous reference level, we abstract from longevity risk. Let $c_{t}$ and $\theta_{t}$ denote the individual's consumption choice and the individual's reference level at adult age $t$, respectively. If consumption is larger (smaller) than the reference level, then the individual experiences a gain (loss). Expected lifetime utility is defined as follows:

$$
U_{0}=\mathbb{E}\left[\int_{0}^{T} e^{-\delta t} u\left(c_{t} ; \theta_{t}\right) \mathrm{d} t\right]
$$

and from Curatola (2017), in a few key aspects. First, habits are not predictable but are hit by unexpected shocks. Second, the habit formation process includes a drift term, reflecting the future state of the world. As a result of this, habits may go down significantly after an increase in current consumption. 
where $T \geq 0$ denotes the adult age at which the individual dies, $\delta \geq 0$ is the subjective rate of time preference, and $u\left(c_{t} ; \theta_{t}\right)$ corresponds to the instantaneous utility function. We assume that instantaneous utility is represented by the canonical two-part power utility function (Tversky and Kahneman (1992)) $]^{7}$

$$
u\left(c_{t} ; \theta_{t}\right)=v\left(c_{t}-\theta_{t}\right)= \begin{cases}\left(c_{t}-\theta_{t}\right)^{\gamma_{G}}, & \text { if } c_{t} \geq \theta_{t} \\ -\kappa\left(\theta_{t}-c_{t}\right)^{\gamma_{L}}, & \text { if } c_{t}<\theta_{t}\end{cases}
$$

Here, $\gamma_{G} \in(0,1)$ and $\gamma_{L}>0$ denote the curvature parameters for gains and losses, respectively, and $\kappa \geq 1$ stands for the loss aversion parameter 8 Figure 1 illustrates the two-part power utility function (2) for two different values of the curvature parameter for losses $\gamma_{L}$. As shown by Figure 1 the two-part power utility function has a kink at the reference level. The kink in the utility function is due to the different treatment of gains and losses.

\section{[Insert Figure 1 near here]}

The two-part power utility function (2) is convex (concave) in the loss domain if the curvature parameter for losses $\gamma_{L}$ is smaller (larger) than unity. Convexity corresponds to risk-loving behavior and concavity to risk-averse behavior 9 Tversky and Kahneman (1992) found experimentally that the utility function is convex in the loss domain. However, this finding is heavily debated in the literature. For instance, Etchart-Vincent (2004) found that a significantly higher proportion of subjects exhibited concavity when facing large losses than when facing small losses; see also Abdellaoui, Vossmann, and Weber (2005), Abdellaoui, Bleichrodt, and Paraschiv (2007) and Booij and van de Kuilen (2009) for further details on this subject matter. Because large (absolute) fluctuations in consumption are not uncommon in a life-cycle setting, we consider both the case of risk-averse and of risk-loving behavior in the loss domain.

A standard assumption of conventional life-cycle models is that relative risk aversion is constant. We do not assume constant relative risk aversion. Rather, the specification in (2) implies that the relative risk aversion function $R\left(c_{t} ; \theta_{t}\right)=-c_{t} u_{c c}\left(c_{t} ; \theta_{t}\right) / u_{c}\left(c_{t} ; \theta_{t}\right)$ depends on

\footnotetext{
${ }^{7}$ This paper considers a partial equilibrium model; that is, we assume that asset prices are exogenously given. For the asset-pricing implications of loss aversion, see, e.g., Shumway (1997), Barberis et al. (2001), Yogo (2008), Andries (2012), Curatola (2015) and Pagel (2017b).

${ }^{\circ}$ There is some debate in the literature on how to define loss aversion. We follow Tversky and Kahneman (1992). Under their specification (2), the disutility of one unit loss is $\kappa$ times larger than the utility of one unit gain. From Section 5 onwards, we assume $\gamma_{L}$ to be at least as large as $\gamma_{G}$, so that $-v(-x)>v(x)$ for all $x>1$. This assumption seems reasonable in a life-cycle setting where the stakes at risk are usually large.

${ }^{9}$ This statement may not be true if probabilities are distorted (see Chew, Karni, and Safra (1987)).
} 
how close current consumption is to the individual's reference level:

$$
R\left(c_{t} ; \theta_{t}\right)= \begin{cases}\left(1-\gamma_{G}\right) \frac{c_{t}}{c_{t}-\theta_{t}}, & \text { if } c_{t}>\theta_{t} \\ +\infty, & \text { if } c_{t}=\theta_{t} \\ -\kappa\left(1-\gamma_{L}\right) \frac{c_{t}}{\theta_{t}-c_{t}}, & \text { if } c_{t}<\theta_{t}\end{cases}
$$

Following the literature on (internal) habit formation (e.g., Constantinides (1990)), we assume that the individual's reference level evolves according to: ${ }^{10} 11$

$$
\mathrm{d} \theta_{t}=\left(\beta c_{t}-\alpha \theta_{t}\right) \mathrm{d} t, \quad \theta_{0} \geq 0 \text { given }
$$

We can explicitly express the reference level $\theta_{t}$ in terms of past consumption choices as follows:

$$
\theta_{t}=e^{-\alpha t} \theta_{0}+\beta \int_{0}^{t} e^{-\alpha(t-s)} c_{s} \mathrm{~d} s .
$$

Here, $\theta_{0}$ denotes the initial reference level, $\alpha \geq 0$ is a persistence (or memory) parameter, and $\beta \geq 0$ controls the relative importance between the initial reference level and the individual's consumption history. When $\alpha$ is small, the effect of current consumption on future reference levels persists for a relatively long time; when $\beta$ increases, consumption history becomes more important. Given fixed $\alpha$, the degree of endogeneity of the reference level increases as $\beta$ increases. The parameter $\beta$ is usually taken to be close to $\alpha$. Indeed, if $\beta$ is close to $\alpha$, then the reference level is given by a weighted average of past consumption choices; see Appendix $D$ for a more formal statement and a derivation of this result.

Finally, we note that our preference model includes several important special (limiting) cases. The habit formation model originally studied by Constantinides (1990) arises as a special case when consumption is larger than the individual's reference level in every state of the economy. If, in addition, the reference level does not depend on the individual's past consumption choices, then the two-part power utility function reduces to a utility function with an exogenous minimum consumption level. Such a utility function has been studied by, for example, Deelstra, Grasselli, and Koehl (2003). The conventional CRRA utility function arises as a special case when the reference level is equal to zero (i.e., when the individual draws utility only from absolute levels of consumption).

\footnotetext{
${ }^{10}$ This reference level specification has been commonly adopted in the habit formation literature, see, e.g., Gomes and Michaelides (2003), Bodie, Detemple, Otruba, and Walter (2004), and Munk (2008).

${ }^{11}$ Köszegi and Rabin (2006, 2007, 2009) and Pagel (2017a) consider a different specification of the individual's reference level. As we show in Section 5 some of our results for optimal consumption behavior are consistent with theirs, despite the differences in specification.
} 


\subsection{Financial Assets and Wealth Accumulation}

This section introduces the financial market. For ease of exposition, we assume in the main text that the market consists of only two assets: a risk-free asset and a risky stock which is traded continuously on the finite time horizon $[0, T]$. Furthermore, investment opportunities are assumed to be constant. Appendix A treats the case of a general financial market with multiple assets and a non-constant investment opportunity set.

Let us denote by $B_{t}$ and $S_{t}$ the risk-free asset price and the risky stock price at adult age $t$, respectively. The risk-free asset price $B_{t}$ evolves as follows:

$$
\frac{\mathrm{d} B_{t}}{B_{t}}=r \mathrm{~d} t, \quad B_{0} \geq 0 \text { given, }
$$

where $r$ denotes the risk-free interest rate at adult age $t$. The risky stock price $S_{t}$ satisfies the following dynamic equation:

$$
\frac{\mathrm{d} S_{t}}{S_{t}}=\mu \mathrm{d} t+\sigma \mathrm{d} Z_{t}, \quad S_{0} \geq 0 \text { given }
$$

where $\mu$ and $\sigma$ represent the expected stock return and the stock return volatility at adult age $t$, respectively, and $\mathrm{d} Z_{t}$ denotes the increment to the Brownian motion that drives the stock return.

Let $M_{t}$ denote the stochastic discount factor at adult age $t$ which is defined as follows:

$$
M_{t}=\exp \left\{-\int_{0}^{t} r \mathrm{~d} s-\int_{0}^{t} \lambda \mathrm{d} Z_{s}-\frac{1}{2} \int_{0}^{t} \lambda^{2} \mathrm{~d} s\right\} .
$$

Here, $\lambda=\sigma^{-1}(\mu-r)$ represents the market price of risk at adult age $t$.

Let $\pi_{t}$ and $W_{t}$ denote the total dollar amounts invested in the risky stock and the individual's total wealth at adult age $t$, respectively. Total wealth $W_{t}$ is made up of both human capital (that is, the discounted value of total lifetime earnings) and financial wealth. Total wealth $W_{t}$ satisfies the following dynamic budget constraint:

$$
\mathrm{d} W_{t}=\left(r W_{t}+\pi_{t} \sigma \lambda-c_{t}\right) \mathrm{d} t+\pi_{t} \sigma \mathrm{d} Z_{t}, \quad W_{0} \geq 0 \text { given }
$$

By integrating the increments $\mathrm{d} W_{t}$, we can show that the individual's total wealth is equal to initial total wealth, plus total trading gains, minus cumulative consumption. The amount of total wealth invested in the risk-free asset at adult age $t$ is given by $W_{t}-\pi_{t}$. We call a consumption and portfolio strategy admissible if total wealth is bounded from below 12

\footnotetext{
${ }^{12}$ This assumption implies that the static budget constraint is also satisfied; see, for example, Karatzas and Shreve (1998, pp. 91-92) for further details.
} 


\subsection{Dynamic Optimization Problem}

The individual maximizes expected lifetime utility subject to the dynamic budget constraint, i.e., the individual faces the following dynamic optimization problem:

$$
\begin{array}{ll}
\max _{c_{t}, \pi_{t}: 0 \leq t \leq T} & \mathbb{E}\left[\int_{0}^{T} e^{-\delta t} v\left(c_{t}-\theta_{t}\right) \mathrm{d} t\right] \\
\text { s.t. } & \mathrm{d} W_{t}=\left(r W_{t}+\pi_{t} \sigma \lambda-c_{t}\right) \mathrm{d} t+\pi_{t} \sigma \mathrm{d} Z_{t}, \\
& \mathrm{~d} \theta_{t}=\left(\beta c_{t}-\alpha \theta_{t}\right) \mathrm{d} t, \\
& \theta_{t}-c_{t} \leq L_{t}^{\max }, \text { for all } t \in[0, T] .
\end{array}
$$

We require that the amount by which consumption $c_{t}$ can fall below the reference level is capped at $L_{t}^{\max } \geq 0{ }^{13}$ The maximum possible loss $L_{t}^{\max }$ is assumed to depend only on adult age $t$ (and not on the state of the economy) ${ }^{14}$

\section{Optimal Policies}

This section presents closed-form expressions for the optimal consumption and portfolio policies. An extensive analysis of the optimal policies is provided in Sections 5 and 6 .

\subsection{Equivalent Optimization Problem}

By virtue of the martingale approach (Pliska (1986), Karatzas, Lehoczky, and Shreve (1987), and Cox and Huang (1989, 1991)), we first transform the dynamic optimization problem (10)

\footnotetext{
${ }^{13}$ Given initial total wealth $W_{0}$, the maximum possible loss $L_{t}^{\max }$ should be such that the set of solutions to the individual's optimization problem 10 is non-empty. We note that the condition $\theta_{t}-c_{t} \leq L_{t}^{\max }$ (for all $t \in[0, T]$ ) is of a primarily technical nature and is moreover only needed in the case of risk-loving behavior in the loss domain. Indeed, in that case, the individual's optimization problem 10 is ill-posed if consumption is not bounded from below (an optimization problem is called ill-posed if its supremum is infinite). One can set $L_{t}^{\max }$ to an arbitrarily large finite number to limit the impact of the lower bound on the optimal policies. For the case of risk-averse behavior in the loss domain, one may take $L_{t}^{\max }$ to be infinity so that the constraint becomes redundant.

${ }^{14}$ We note that, in the case of an endogenous reference level, consumption can, in principle, fall below zero. It can, however, never fall below the reference level by more than $L_{t}$ (i.e., $c_{t}$ always exceeds $\theta_{t}-L_{t}$ ). For our benchmark parameter values (see Section 5.3), we find that the (unconditional) probability of consumption being non-positive ranges from $0.00 \%$ to $0.12 \%$, depending on the individual's age. We have also computed the discounted value of non-positive optimal consumption, i.e., $\mathbb{E}\left[\int_{0}^{T} M_{t} \min \left(0, c_{t}^{*}\right) \mathrm{d} t\right]$. This number is equal to $1.98 \%$ of initial wealth for our benchmark case. Based on these numbers, we conclude that the impact of non-positive consumption on the optimal policies is very small.
} 
into the following equivalent static variational problem ${ }^{15}$

$$
\begin{array}{ll}
\max _{c_{t}: 0 \leq t \leq T} & \mathbb{E}\left[\int_{0}^{T} e^{-\delta t} v\left(c_{t}-\theta_{t}\right) \mathrm{d} t\right], \\
\text { s.t. } & \mathbb{E}\left[\int_{0}^{T} M_{t} c_{t} \mathrm{~d} t\right] \leq W_{0}, \\
& \mathrm{~d} \theta_{t}=\left(\beta c_{t}-\alpha \theta_{t}\right) \mathrm{d} t, \\
& \theta_{t}-c_{t} \leq L_{t}^{\max }, \text { for all } t \in[0, T] .
\end{array}
$$

After we have solved for the optimal consumption strategy $c_{t}^{*}$, we determine the optimal portfolio strategy $\pi_{t}^{*}$ using replication arguments. Sections 4.2 and 4.3 present the optimal consumption and portfolio policies, respectively.

\subsection{Optimal Consumption Choice}

Theorem 4.1 presents the individual's optimal consumption choice $c_{t}^{*}$.

Theorem 4.1 (OPTIMAL COnSUmption ChOICE). Consider an individual with the two-part power utility function (2) and reference level specification (5) who solves the optimization problem (11). Denote by $\theta_{t}^{*}$ the individual's optimal reference level at adult age $t$ implied by substituting the optimal past consumption choices into (5), by y the Lagrange multiplier associated with the static budget constraint in (11), and by $\widehat{M}_{t}=M_{t}\left(1+\beta F_{t}\right)$ the so-called dual stochastic discount factor at adult age $t$. Here, $F_{t}$ denotes the price at adult age $t$ of a bond with coupon process $\left\{e^{-(\alpha-\beta)(s-t)}\right\}_{s \geq t}$, i.e. 16

$$
F_{t}=\mathbb{E}_{t}\left[\int_{t}^{T} \frac{M_{s}}{M_{t}} e^{-(\alpha-\beta)(s-t)} \mathrm{d} s\right]
$$

Then:

(i) If the individual is risk averse in the loss domain, the optimal consumption choice at adult age $t$ is given by

$$
c_{t}^{*}= \begin{cases}\theta_{t}^{*}+\left(\frac{y e^{\delta t} \widehat{M}_{t}}{\gamma_{G} \widehat{M}_{0}}\right)^{\frac{1}{\gamma_{G}-1}}, & \text { if } \frac{\widehat{M}_{t}}{\widehat{M}_{0}} \leq \xi_{t} \\ \theta_{t}^{*}-\min \left\{\left(\frac{y e^{\delta t} \widehat{M}_{t}}{\kappa \gamma_{L} \widehat{M}_{0}}\right)^{\frac{1}{\gamma_{L}-1}}, L_{t}^{\max }\right\}, & \text { if } \frac{\widehat{M}_{t}}{\widehat{M}_{0}}>\xi_{t} .\end{cases}
$$

\footnotetext{
${ }^{15}$ Note that terminal wealth $W_{T}$ is zero by construction.

${ }^{16}$ Note that under the assumption of a constant investment opportunity set, $F_{t}$ can be computed explicitly, i.e.,

$$
F_{t}=\frac{1}{r+\alpha-\beta}\left[1-e^{-(r+\alpha-\beta)(T-t)}\right]
$$
}


Here, the threshold $\xi_{t}$ is implicitly defined as follows:

$$
\begin{aligned}
0= & e^{-\delta t}\left(1-\gamma_{G}\right)\left(\frac{y e^{\delta t} \xi_{t}}{\gamma_{G}}\right)^{\frac{\gamma_{G}}{\gamma_{G}-1}}+e^{-\delta t} \kappa \min \left\{\left(\frac{y e^{\delta t} \xi_{t}}{\kappa \gamma_{L}}\right)^{\frac{1}{\gamma_{L}-1}}, L_{t}^{\max }\right\}^{\gamma_{L}} \\
& -y \xi_{t} \min \left\{\left(\frac{y e^{\delta t} \xi_{t}}{\kappa \gamma_{L}}\right)^{\frac{1}{\gamma_{L}-1}}, L_{t}^{\max }\right\} .
\end{aligned}
$$

(ii) If the individual is risk loving in the loss domain, the optimal consumption choice at adult age $t$ is given by

$$
c_{t}^{*}= \begin{cases}\theta_{t}^{*}+\left(\frac{y e^{\delta t} \widehat{M}_{t}}{\gamma_{G} \widehat{M}_{0}}\right)^{\frac{1}{\gamma_{G}-1}}, & \text { if } \frac{\widehat{M}_{t}}{\widehat{M}_{0}} \leq \zeta_{t} \\ \theta_{t}^{*}-L_{t}^{\max }, & \text { if } \frac{\widehat{M}_{t}}{\widehat{M}_{0}}>\zeta_{t} .\end{cases}
$$

Here, the threshold $\zeta_{t}$ is implicitly defined as follows:

$$
0=e^{-\delta t}\left(1-\gamma_{G}\right)\left(\frac{y e^{\delta t} \zeta_{t}}{\gamma_{G}}\right)^{\frac{\gamma_{G}}{\gamma_{G}-1}}+e^{-\delta t} \kappa\left(L_{t}^{\max }\right)^{\gamma_{L}}-y \zeta_{t} L_{t}^{\max } .
$$

The Lagrange multiplier $y$ is chosen such that the static budget constraint in (11) holds with equality.

Note first that the gain given by $\max \left(0, c_{t}^{*}-\theta_{t}^{*}\right)$ with $c_{t}^{*}$ as in 13$)$ and 15 has a similar representation as the familiar Merton consumption strategy. Here, the value of the dual stochastic discount factor $\widehat{M}_{t}$ represents the current state of the economy; it differs from the value of the ordinary stochastic discount factor $M_{t}$ (see (8)), to which it is proportional, due to the endogeneity in the individual's reference level as captured through the factor $1+\beta F_{t}$. The larger the degree of endogeneity $\beta$, the more $\widehat{M}_{t}$ will differ from $M_{t}$. In the special case where $\beta=0, \widehat{M}_{t}$ coincides with $M_{t}$. Next, based on how large $\widehat{M}_{t}$ is compared to the threshold $\xi_{t}$ (risk-averse behavior in the loss domain) or the threshold $\zeta_{t}$ (risk-loving behavior in the loss domain), both depending on the model parameters, the individual decides whether to consume more or less than his current reference level. Finally, in the risk-averse case, the loss given by $-\min \left(0, c_{t}^{*}-\theta_{t}^{*}\right)$ with $c_{t}^{*}$ as in 13$)$ resembles the familiar Merton consumption strategy (up to the loss $L_{t}^{\max }$ ), while in the risk-loving case, the loss always equals $L_{t}^{\max }$.

We give the full proof of Theorem 4.1 in Appendix C here, we only sketch the intuition behind the proof. First, by invoking the solution technique of Schroder and Skiadas (2002), we transform the individual's optimization problem (11) into a dual optimization problem without endogenous updating of the individual's reference level; see Appendix B for further details. The dual optimization problem is defined in terms of dual (or surplus) consumption $\widehat{c}_{t}=c_{t}-\theta_{t}$. Next, we solve the dual optimization problem in a dual financial market that is characterized by the 
dual risk-free interest rate, the dual stock return volatility, the dual stochastic discount factor, and the dual market price of risk; see Appendix B for the definitions of these quantities. The dual optimization problem is solved by using convex duality (or martingale) techniques, and by using the techniques proposed by Basak and Shapiro (2001) (see also Berkelaar et al. (2004)) to deal with pseudo-concavity and non-differentiability aspects of the dual optimization problem. We suitably adapt the latter techniques to our setting with intertemporal consumption. The central step is to split the individual's optimization (dual, in our case) problem into two separate problems: a gain part problem and a loss part problem. The optimal solution corresponding to each problem is a local maximum of the dual optimization problem. The global maximum of the dual problem is found by comparing the two local maxima; see Appendix C for more details. Finally, using Proposition 1 in Appendix B, we convert the optimal dual consumption choice $\widehat{c}_{t}^{*}$ back into the optimal (primal) consumption choice $c_{t}^{*}$.

\subsection{Optimal Portfolio Choice}

Theorem 4.2 presents the optimal portfolio choice $\pi_{t}^{*}$.

Theorem 4.2 (OPTIMAL PORTFOLIO CHOICE). Consider an individual with the two-part power utility function (2) and reference level specification (5) who solves the optimization problem (11). Let $F_{t}$ be defined by (12). Then the optimal portfolio strategy at adult age $t$ is given by

$$
\pi_{t}^{*}=\widehat{\pi}_{t}^{*}+\beta F_{t} \widehat{\pi}_{t}^{*}
$$

where $\widehat{\pi}_{t}^{*}$ denotes the optimal dual portfolio strategy at adult age $t$ and is defined as follows:

$$
\widehat{\pi}_{t}^{*}= \begin{cases}A_{t} \widehat{W}_{t}^{G *}+B_{t} \widehat{W}_{t}^{L *}, & \text { if } \gamma_{L}>1 \\ C_{t} \widehat{W}_{t}^{G *}+D_{t} \widehat{W}_{t}^{L *}, & \text { if } \gamma_{L} \in(0,1] .\end{cases}
$$

Here, wealth $W_{t}^{*}$ to finance future optimal consumption is decomposed into $\widehat{W}_{t}^{G *} \geq 0$ and $-\widehat{W}_{t}^{L *} \leq 0$, which denote the present value of future optimal gains $\left\{\max \left(0, c_{s}^{*}-\theta_{s}^{*}\right)\right\}_{s \geq t}$ and the present value of future optimal losses $\left\{\min \left(0, c_{s}^{*}-\theta_{s}^{*}\right)\right\}_{s>t}$, respectively, and a residual which finances future optimal reference levels $\left\{\theta_{s}^{*}\right\}_{s \geq t}$. The explicit expressions for $\widehat{W}_{t}^{G *}$ and $\widehat{W}_{t}^{L *}$ are given in Appendix C; see (58) and (59) for the case of risk-averse behavior in the loss domain, and (69) and (70) for the case of risk-loving behavior in the loss domain. The coefficients $A_{t}$ $\left(\gamma_{L}>1\right)$ and $C_{t}\left(\gamma_{L} \in(0,1]\right)$ denote the shares of $\widehat{W}_{t}^{G *}$ invested in the risky stock, while $B_{t}$ $\left(\gamma_{L}>1\right)$ and $D_{t}\left(\gamma_{L} \in(0,1]\right)$ represent the shares of $\widehat{W}_{t}^{L *}$ invested in the risky stock. They are also defined in Appendix C; see (76)-(79).

Theorem 4.2 shows that the individual's optimal portfolio strategy $\pi_{t}^{*}$ can be decomposed into several terms. If the reference level is equal to zero (i.e., $\beta=0$ and $\widehat{W}_{t}^{L *}=0$ ), optimal 
total wealth $W_{t}^{*}$ coincides with the present value of future optimal gains $\widehat{W}_{t}^{G *}$. In that case, the optimal portfolio strategy $\pi_{t}^{*}$ reduces to the term $\left[\sigma\left(1-\gamma_{G}\right)\right]^{-1} \lambda \widehat{W}_{t}^{G *}=\left[\sigma\left(1-\gamma_{G}\right)\right]^{-1} \lambda \widehat{W}_{t}^{*}$ which corresponds to the optimal portfolio strategy of an individual with conventional CRRA utility, who invests a constant proportion of total wealth in risky stocks.

The optimal portfolio strategy of an individual whose preferences are represented by the difference internal habit model (Constantinides (1990)) also arises as a special case of Theorem 4.2 (i.e., $\beta \neq 0$ and $\widehat{W}_{t}^{L *}=0$ ). An individual with such preferences divides his total wealth into two parts. He uses $\widehat{W}_{t}^{G *}$ to finance future optimal gains, and $W_{t}^{*}-\widehat{W}_{t}^{G *}$ to finance future optimal reference levels. Compared to conventional CRRA utility, the optimal portfolio strategy under the difference internal habit model differs in two ways. First, relative risk aversion is not constant under the difference internal habit model: the individual becomes more risk averse as consumption approaches the reference level. This fact leads to a state-dependent (rather than constant) optimal portfolio weight. The coefficients $A_{t}$ (risk-averse behavior in the loss domain) and $C_{t}$ (risk-loving behavior in the loss domain) capture this effect ${ }^{17}$ Second, habit formation leads to an optimal portfolio strategy in which the exposure to the risky stock decreases, on average, as the individual grows older. This effect is captured by the term $\beta F_{t} \widehat{\pi}_{t}^{*}$ in (17).

If the individual views the reference level as a standard-of-living (i.e., $\beta \neq 0$ and $\widehat{W}_{t}^{L *} \neq 0$ ) rather than as a minimum subsistence level (as under the difference internal habit model), so that consumption can fall below the reference level, then the individual divides his total wealth into three (and not two) parts. He uses $\widehat{W}_{t}^{G *}$ to finance future optimal gains, $-\widehat{W}_{t}^{L *}$ to finance future optimal losses, and $W_{t}^{*}-\widehat{W}_{t}^{G *}+\widehat{W}_{t}^{L *}$ to finance future optimal reference levels. The coefficients $B_{t} \neq A_{t}$ (risk-averse behavior in the loss domain) and $D_{t} \neq C_{t}$ (risk-loving behavior in the loss domain) arise because the individual's attitude towards risk is very different in the loss domain than in the gain domain (see equation (3p).

\section{Main Implications}

This section explores our main implications. Section 5.1 outlines the setting used throughout the analysis. Section 5.2 analyzes the optimal consumption and portfolio choice of a loss-averse individual with a constant reference level. Section 5.3 explores how endogenous updating of the reference level affects the individual's behavior. Section 5.4 investigates the existing empirical literature in relation to our main findings. Finally, Section 5.5 explains how we can incorporate probability weighting, which is another main ingredient of prospect theory, into our consumption and investment model.

\footnotetext{
${ }^{17}$ We note that under the difference internal habit model, the coefficients $A_{t}$ and $C_{t}$ coincide.
} 


\subsection{Setting}

We consider a loss-averse individual who works from age 25 to his retirement age 65 . His salary equals $\$ 50,000$ per year, which roughly corresponds to the median household income in the U.S. in 2014. Human capital at age 25 is given by the discounted value of total lifetime earnings. Financial wealth at age 25 is zero. We assume that the individual passes away at age 85 . In each period, the individual decides how much of his total wealth (i.e., the sum of human capital and financial wealth) to consume and how much to invest in the risk-free asset and the risky stock. The return on the risk-free asset is constant and set at $1 \%$. The equity risk premium and the stock return volatility are constant as well. They are set at $4 \%$ and $20 \%$, respectively. These parameter values coincide with those used by Gomes, Kotlikoff, and Viceira (2008).

Estimates of the individual's preference parameters differ widely across studies (see for an overview Abdellaoui et al. (2007) and Booij, van Praag, and van de Kuilen (2010)). We set the loss aversion parameter $\kappa$ at 2 and the curvature parameter for gains $\gamma_{G}$ at 0.4 . The curvature parameter for losses $\gamma_{L}$ is set equal to either 1.3 (risk-averse behavior in the loss domain) or 0.95 (risk-loving behavior in the loss domain). These parameter values generate realistic consumption and portfolio policies. In Section 6, we explore in detail the roles of the individual's preference parameters in determining the optimal consumption and portfolio policies ${ }^{18}$ The subjective rate of time preference $\delta$ equals $4 \% 19$ Our main implications remain qualitatively unchanged if we vary the values of the return and preference parameters within reasonable limits.

\subsection{A Loss-Averse Individual with a Constant Reference Level}

This section considers the optimal consumption and portfolio choice of a loss-averse individual with a constant reference level; that is, $\theta_{t}=\theta$. Inspired by Barberis et al. (2001), we set the reference level equal to the constant consumption level the individual would obtain if, in every period, his total wealth were invested only in the risk-free asset ${ }^{20}$

To understand the optimal behavior of a loss-averse individual, we first recall the behavior of an individual with conventional CRRA preferences (henceforth also referred to as a CRRA individual). When the stock price falls, a CRRA individual decreases his consumption level proportionally to the decline in the stock price. How close current consumption is to a reference level plays no role. As a result, the consumption-to-total-wealth ratio (i.e., the share of total wealth consumed) and the portfolio strategy (i.e., the share of total wealth invested in the risky stock) are constant across states of the economy: a CRRA individual does not adjust his consumption-to-total-wealth ratio and portfolio strategy in response to a stock price movement.

\footnotetext{
${ }^{18}$ Section 6 also studies the relationship between the two-part power utility function and the CRRA utility function.

${ }^{19}$ According to Samwick (1998), median rates of time preference for U.S. households are between $3 \%$ and $4 \%$.

${ }^{20}$ Barberis et al. (2001) argue that the risk-free interest rate serves as a natural benchmark for evaluating gains and losses.
} 
Only age and attitude towards risk and time affect the optimal strategies. In the analysis that follows, we compare our findings to this conventional wisdom.

Contrary to a CRRA individual, a loss-averse individual derives utility from the difference between consumption and his reference level: consuming below the reference level is relatively painful due to loss aversion. A loss-averse individual thus takes his reference level into account when making optimal consumption and portfolio decisions, leading to a state-dependent (rather than constant) optimal consumption-to-total-wealth ratio and portfolio strategy. Figure 2 shows how the optimal current choice of a 65 -year-old loss-averse individua ${ }^{21}$ depends on the the past stock return. The left panels assume risk-averse behavior in the loss domain, whereas the right panels assume risk-loving behavior in the loss domain. The individual classifies past stock returns into three categories: (i) a normal past stock return in which current consumption is at (or slightly above) the reference level; (ii) a good past stock return in which current consumption is substantially above the reference level; and (iii) a bad past stock return in which current consumption is below the reference level. The following sections describe the individual's behavior for each category.

\section{[Insert Figure 2 and Table 1 near here]}

\subsubsection{Normal Past Stock Return}

In case of a normal past stock return, the individual consumes at, or slightly more than, his reference level; see the solid line in Figures 2(a) and (b). A loss-averse individual thus adopts a self-insurance strategy to minimize the risk of a loss ${ }^{22}$ The individual finances the self-insurance strategy by giving up some of the potential on the upside. In contrast to Basak and Shapiro (2001), our model generates a self-insurance strategy without imposing an exogenous constraint (e.g., Value-at-Risk constraint) on consumption.

As illustrated by the solid line in Figures $2(\mathrm{c})$ and (d), in case of a normal past stock return, the consumption-to-total-wealth ratio increases as the stock price declines: a loss-averse individual does not, or only to a very limited extent, adjust his current level of consumption in response to a change in total wealth. By delaying painful cuts in current consumption following a negative wealth shock, the individual avoids an immediate loss. Intuitively, he prefers an uncertain loss tomorrow over a certain loss today. The flipside of protecting current consumption is, however, that an improvement in the individual's financial position does not directly translate into a higher level of current consumption; rather, it goes into a precautionary savings fund to support consumption in the event of an adverse shock. Köszegi and Rabin (2009) and Pagel (2017a) also find that a loss-averse individual holds a (first-order) precautionary savings motive.

\footnotetext{
${ }^{21}$ Table 1 gives a number of properties of the optimal consumption and portfolio policies at other ages.

${ }^{22}$ The individual optimally desires to consume more than his reference level; only under genuinely adverse circumstances, the individual consumes below his reference level (see also Section 5.2.3).
} 
To avoid an immediate loss, a loss-averse individual implements a (very) conservative portfolio strategy in case of a normal past stock return. He typically invests less in the risky stock than a CRRA individual; see Figures $2(\mathrm{e})$ and $(\mathrm{f}){ }^{23}$ Generally speaking, the closer current and expected future consumption levels are to the reference level, the lower the individual's willingness to invest in the risky stock. Indeed, the relative risk aversion function (as defined in (3p) increases sharply as consumption approaches the reference level. Relative risk aversion is thus not constant, as is assumed in many life-cycle models, but rather depends on how close current and expected future consumption levels are to the reference level.

Finally, we note that relative risk aversion is highest if $c_{t}=\theta_{t}$. Based on this observation, one might expect that the portfolio strategy attains a (local) minimum in the state of the economy where consumption is closest to the reference level. We find, however, that in scenarios where consumption is slightly more than the reference level, the portfolio strategy is, in fact, a decreasing function of the past stock return; see Figures 2(e) and (f). The reason is that in these scenarios, although current consumption exceeds the current reference level, the probability that future levels of relative risk aversion are large is relatively high. Because the portfolio strategy takes into account not just the individual's current level of relative risk aversion but also (probabilities of) future levels of relative risk aversion, the portfolio strategy attains a (local) minimum in a scenario where consumption is slightly above the reference level.

\subsubsection{Good Past Stock Return}

In case of a good past stock return, the individual consumes substantially more than the reference level; see the solid line in Figures [2(a) and (b). In that case, the consumption-to-total-wealth ratio decreases (rather than increases as in Section 5.2.1 above) when the stock price declines, as depicted by the solid line in Figures 22(c) and (d). Consumption thus over-responds (rather than under-responds) to a change in total wealth. Indeed, in case of a good past stock return, the individual can fully absorb a wealth shock into current consumption without causing current consumption to fall below the reference level. In fact, since the individual is relatively rich (compared to the costs of financing future reference levels), he increases precautionary savings following a negative wealth shock. Because future consumption levels will likely exceed the reference level, a loss-averse individual has no reason to adopt a (very) conservative portfolio strategy in case of a good past stock return. He may even invest more in the risky stock than a CRRA individual; see Figures 2(e) and (f).

\footnotetext{
${ }^{23}$ If the loss aversion index $\kappa$ is sufficiently high, then the optimal portfolio weight takes on very low values in some scenarios. The question becomes then whether or not in practice the individual should exit the stock market in these scenarios. Exiting the stock market might be optimal in an economy with fixed stock market participation costs.
} 


\subsubsection{Bad Past Stock Return}

In case of a bad past stock return, the individual consumes less than the reference level; see the solid line in Figures 2(a) and (b). As these figures illustrate, the optimal consumption policy differs between the case of risk-loving behavior in the loss domain and the case of risk-averse behavior in the loss domain.

If the individual is risk loving in the loss domain (i.e., $\gamma_{L}$ is smaller than unity), then the individual follows a gambling policy. In case of a normal and a good past stock return, the individual consumes more than the reference level, while in case of a bad past stock return, he accepts a fixed loss of $L_{t}^{\max }$. Because a stock price movement leaves current consumption unaffected in case of a bad past stock return, the consumption-to-total-wealth ratio increases as the stock price declines, as illustrated by the solid line in Figure 2(d). To finance the minimum consumption level $\theta-L_{t}^{\max }$, the individual invests a relatively large share of his total wealth in the risk-free asset; see the solid line in Figure 2(f). Berkelaar et al. (2004), Jin and Zhou (2008) and He and Zhou (2011, 2016) also find that a loss-averse individual who is risk loving in the loss domain adopts a gambling investment policy.

If the individual is risk averse in the loss domain (i.e., $\gamma_{L}$ is larger than unity), consumption is not constant, but rather varies with past stock performance. As a result, the individual has a relatively large exposure to the risky stock, as shown by the solid line in Figure 2(e) ${ }^{24}$ By adopting a relatively aggressive portfolio strategy, the individual increases the chance of a future gain. Although current consumption depends on past stock performance, a wealth shock is typically not fully reflected into current consumption. Indeed, as illustrated by the solid line in Figure 2(c), the consumption-to-total-wealth ratio typically increases as the stock price declines. By not fully absorbing negative wealth shocks into current consumption, the individual avoids big reductions in current consumption. The flipside of this behavior is, however, that the individual benefits only partially from an increase in total wealth.

\subsection{A Loss-Averse Individual with an Endogenous Reference Level}

This section considers a loss-averse individual who endogenously updates his reference level over time. We choose the values of the preference parameters describing the reference level dynamics (4) such that i) the reference level is given by a weighted average of past consumption choices, and ii) the most recent 5 years of consumption contribute at least $80 \%$ of all past consumption to the reference level $\left[{ }^{25}\right.$ In addition, we choose the loss aversion index $\kappa$ and the curvature parameter for gains $\gamma_{G}$ such that the individual's median level of relative risk aversion at age 50 will remain the same, to facilitate the comparison between a loss-averse individual with a

\footnotetext{
${ }^{24}$ In order to finance the minimum consumption level $\theta-L_{t}^{\max }$, the optimal portfolio policy eventually converges to zero as the past stock return goes to $-100 \%$, similar to the case of risk-loving behavior in the loss domain.

${ }^{25}$ These two criteria fully characterize the preference parameters $\alpha$ and $\beta$ in equation (4). More specifically, we find $\alpha=\beta=0.3$.
} 
constant reference level and a loss-averse individual with an endogenous reference level. We find $\kappa=5$ and $\gamma_{G}=0.2$. The initial reference level $\theta_{0}$ has the same value as in Section 5.2 .

A loss-averse individual with an endogenous reference level has a strong incentive to postpone painful cuts in current consumption following a negative wealth shock. Because the individual is loss-averse relative to an endogenous reference level, a reduction in future consumption levels is felt less heavily than a reduction in his current consumption level. Indeed, when the individual's total wealth decreases as a result of a poor stock return, future reference levels decrease as well, while his current reference level remains unaffected.

Figure 3 shows the impact of a $10 \%$ drop in the individual's total wealth at age 45 on expected consumption for medium-wealth individuals (panels (a) and (b)], high-wealth individuals (panels (c) and (d) $)$, and low-wealth individuals (panels (e) and (f)). As shown by the dashed lines in Figure 3, a CRRA individual reduces current and expected future consumption levels by the same percentage (i.e., 10\%), irrespective of the current value of his total wealth. By contrast, a loss-averse individual absorbs a wealth shock based on how much total wealth he possesses relative to the costs of financing future reference levels.

Furthermore, we have also computed the shock absorbing mechanism, which describes how changes in past consumption impact future consumption, for other values of $\kappa, \gamma_{G}$ and $\gamma_{L}$. It turns out that the mechanism is quite insensitive to a change in these parameter values. However, the shock absorbing mechanism does vary with changes in $\alpha$ and $\beta$. Table 2 shows, for various values of $\alpha$ and $\beta$, the impact of a $10 \%$ drop in the individual's total wealth on expected future consumption. This table clearly shows that the higher $\alpha$ and $\beta$ are, the larger the individual's tendency to postpone reductions in current consumption (i.e., the higher the degree of excess smoothness in consumption).

\section{[Insert Figure 3 and Table 2 near here]}

Panels (a) and (b) illustrate the optimal behavior of medium-wealth individuals (i.e., the individual's total wealth at age 45 is equal to the costs of financing a consumption stream that is equal to the reference level at age 45). As shown by the dash-dotted line, to avoid that current consumption falls below the reference level, an individual with a constant reference level postpones cuts in current consumption; see also Section 5.2.1. A loss-averse individual with an endogenous reference level has an even stronger incentive to postpone cuts in current consumption, as shown by the solid line. He postpones cuts in current consumption not only because he wants to avoid that current consumption falls below the reference level, but also because with a decreasing endogenous reference level, a reduction in future consumption levels is less painful than a reduction in his current consumption level ${ }^{26}$ Current consumption is thus reduced by much less than $10 \%$. Because the wealth shock is not fully reflected into current consumption, expected consumption at ages 68 to 85 is reduced by more than $10 \%$.

\footnotetext{
${ }^{26}$ Appendix E shows this intuition formally.
} 
Consumption is thus excessively smooth and excessively sensitive. Kőszegi and Rabin (2009) and Pagel (2017a), within a discrete-time consumption model that has a forward-looking rather than a backward-looking reference level specification, also find that the consumption stream of a loss-averse individual satisfies these two empirically observed properties.

Panels (c) and (d) illustrate the optimal behavior of high-wealth individuals (i.e., the individual's total wealth at age 45 is twice the costs of financing a consumption stream that is equal to the reference level at age 45). As shown by the dash-dotted line, an individual with a constant reference level no longer has an incentive to postpone cuts in current consumption; see also Section 5.2.2. Indeed, full absorption of wealth shocks does not lead to an immediate loss. He even increases savings following a negative wealth shock so as to prepare for bad times. By contrast, a loss-averse individual with an endogenous reference level still postpones cuts in current consumption following a wealth shock, as shown by the solid line. Although there is no need to protect current consumption, a reduction in future consumption levels is still felt less heavily than a reduction in his current consumption level.

Panels (e) and (f) display the optimal behavior of low-wealth individuals (i.e., the individual's total wealth at age 45 is $85 \%$ of the costs of financing a consumption stream that is equal to the reference level at age 45). As shown by the dash-dotted line, a low-wealth individual with a constant reference level gradually adjusts current consumption in response to a wealth shock. However, this individual absorbs a wealth shock much quicker into consumption than a medium-wealth individual with a constant reference level. The main driver for this is that the low-wealth individual is primarily concerned about avoiding large losses in current consumption rather than to prevent consumption from falling below the reference level as is the prime concern of the medium-wealth individual. A low-wealth individual with an endogenous reference level has a stronger incentive to postpone cuts in current consumption following a negative wealth shock than a low-wealth individual with a constant reference level; see the solid line. Indeed, a reduction in current consumption causes a relatively large loss, while, with a decreasing endogenous reference level, a reduction in future consumption causes potentially a smaller loss.

While the optimal portfolio profile under an endogenous reference level has the same shape as in Section 5.2 , it is typically shifted upwards, everything else being equal. Indeed, because the reference level is not constant but rather depends on past consumption choices, the individual can typically afford to adopt a less conservative portfolio strategy. Furthermore, the individual follows a life-cycle investment strategy (i.e., the share of total wealth invested in the risky stock decreases, on average, over the life-cycle), even in the absence of human wealth; see the solid line in Figures $4(\mathrm{a})$ and (b) ${ }^{27}$ Intuitively, as the individual grows older, he has less time to

\footnotetext{
27 Bodie et al. (1992) give a justification for adopting a life-cycle investment strategy based on human capital considerations. If human capital is risk-free, individuals implicitly hold a risk-free asset. To offset this implicit risk-free asset holding, financial wealth should be tilted toward risky assets. As the share of human capital in total wealth decreases from one to zero during the working period, the optimal share of financial wealth invested
} 
absorb wealth shocks (i.e., his intertemporal rate of substitution decreases over time). Hence, to maintain an excessively smooth and excessively sensitive consumption stream, he reduces the exposure to the stock over the course of his life. We note that a CRRA individual and a loss-averse individual with a constant reference level do not adopt a life-cycle investment strategy in terms of total wealth; see the dash-dotted line in Figures $4(\mathrm{a})$ and (b). As a result, in terms of financial wealth, a loss-averse individual with an endogenous reference level has a stronger preference for a life-cycle investment strategy than a CRRA individual or a loss-averse individual with a constant reference level; see Figures $4 \mid(\mathrm{c})$ and $(\mathrm{d}){ }^{28}$ In other words, the portfolio strategy of an individual with an endogenous reference level is more sensitive to age than the portfolio strategy of an individual with a constant reference level.

\section{[Insert Figure 4 near here]}

Furthermore, we have also computed the median share of total wealth invested in the risky stock for other values of $\kappa, \gamma_{G}$ and $\gamma_{L}$. We find that a change in these parameter values hardly affects the shape of the portfolio profile. It only affects the average level of risk taking because relative risk aversion changes. A change in $\alpha$ and $\beta$ does have an impact on the shape of the portfolio profile, but overall the portfolio share remains decreasing with age. This can be concluded from Figure 5 which shows, for various values of $\alpha$ and $\beta$, the median share of total wealth invested in the risky stock over the life cycle.

\section{[Insert Figure 5 near here]}

Finally, we note that an individual with preferences that comply with the difference internal habit model (Constantinides (1990)) also implements a life-cycle investment strategy. However, such an individual typically invests more in risky stocks than a loss-averse individual with an endogenous reference level 29 Indeed, an individual whose preferences are described by the difference internal habit model views the reference level as a minimum subsistence level rather than as a standard-of-living, so that $\widehat{W}_{t}^{G *}$ (i.e., the present value to finance future optimal gains) will be relatively large. As a result, an individual with an endogenous minimum subsistence level pursues a relatively risky investment strategy; see also Theorem 4.2 .

in risk-free assets increases over the life-cycle.

${ }^{28}$ The optimal share of financial wealth invested in the risky stock, $f_{t}^{*}$, can be computed as follows:

$$
f_{t}^{*}=\frac{\pi_{t}^{*}}{W_{t}^{*}-H_{t}}
$$

where $H_{t}$ represents human capital (i.e., the value at adult age $t$ of future labor earnings). For illustration purposes, Figures $4(\mathrm{e})$ and (f) show how median total wealth, median financial wealth, and human capital evolve over the individual's life cycle.

${ }^{29}$ Here, we implicitly assume that the curvature parameter for gains is the same for both individuals. 


\subsection{Review of Existing Empirical Literature in Relation to our Findings}

Our consumption and investment model with loss aversion and an endogenous reference level generates a number of implications that can be tested using data on consumption expenditures and portfolio holdings. This section discusses the existing empirical literature in relation to our model implications.

A first testable implication of our model is that consumption is excessively smooth (i.e., under-responds to wealth shocks) and excessively sensitive (i.e., past wealth shocks predict current consumption growth), and particularly so with a high degree of endogeneity of the reference level. This finding holds for a wide range of parameter values; see Section 5.3 and Table 2. We can test our first model implication, and estimate the degree of excess smoothness and excess sensitivity, by regressing changes in current consumption on changes in current total wealth levels and changes in past total wealth levels.

To illustrate the connection between this model implication and empirical facts, we use simulated consumption and wealth data and run the following regression model:

$$
\Delta \log c_{t}^{*}=\sum_{i=1}^{5} \beta_{i} \Delta \log W_{t+1-i}^{*}+\epsilon_{t}
$$

where $\Delta \log c_{t}^{*}$ and $\Delta \log W_{t}^{*}$ denote the changes in $\log$ (demeaned) optimal consumption and $\log$ (demeaned) optimal total wealth between adult age $t-1$ and adult age $t$, respectively, and $\epsilon_{t}$ represents the error term at adult age $t$. If $0 \leq \beta_{1}<1$, consumption is excessively smooth: it responds less than one-to-one to a change in wealth. If $\beta_{i}>0$ for some $i>1$, consumption is excessively sensitive: past wealth shocks have predictive power for current consumption growth. We assume that the individual is currently aged 65 . Table 4 reports our results. The regression analysis confirms that a loss-averse individual with an endogenous reference level exhibits excess smoothness and excess sensitivity, as $0 \leq \widehat{\beta}_{1}<1$ and $\widehat{\beta}_{i}>0(i>1)$ for all sets of parameters we consider. By contrast, the CRRA individual has $\widehat{\beta}_{1}=1$ and $\widehat{\beta}_{i}=0$ for every $i>1$. Furthermore, we observe from Table 4 that as the degree of endogeneity of the reference level increases, consumption becomes more excessively smooth (i.e., $\widehat{\beta}_{1}$ decreases) and more excessively sensitive (i.e., $\widehat{\beta}_{2}, \ldots, \widehat{\beta}_{5}$ increase). Numerous empirical studies find pronounced evidence of excess smoothness and excess sensitivity in aggregate consumption data; see, e.g., Flavin (1985), Deaton (1987), and Campbell and Deaton (1989). Furthermore, many annuity providers nowadays offer variable annuity type products in which payouts respond sluggishly, rather than directly, to stock return shocks ${ }^{30}$ Our results, in particular those with both loss aversion and endogenous updating in Section 5.3, thus provide a preference-based justification for the existence of these (new) type of annuities.

\footnotetext{
${ }^{30}$ For more details on these (new) type of annuities, see, e.g., Guillén, Jørgensen, and Nielsen (2006), Jørgensen and Linnemann (2011), Guillén, Nielsen, Pérez-Marín, and Petersen (2013), Linnemann, Bruhn, and Steffensen (2014) and Maurer, Mitchell, Rogalla, and Siegelin|(2016).
} 


\section{[Insert Table 4 near here]}

However, if individuals are heterogenous, properties of aggregate consumption data may not be observed in consumption data at the micro-level. Interestingly, our results reveal that the degree of excess smoothness and excess sensitivity in consumption varies considerably across individuals. In particular, we find that the consumption stream of a medium-wealth individual is typically smoother and more sensitive than the consumption stream of a low-wealth and, especially, a high-wealth individual; see Section 5.3. This hypothesis can also be tested in the data. Existing empirical studies already find some evidence in favor of this hypothesis; see, e.g., Dynan, Skinner, and Zeldes (2004), and Johnson, Parker, and Souleles (2006). More specifically, our comparative statics results are in line with the existing literature which finds that the marginal propensity to consume, measuring the change in consumption due to a change in income, is smaller for medium-wealth individuals than for low-wealth and high-wealth individuals.

Our results on portfolio choice show that the degree of risk-taking depends on the state of the economy and the loss aversion parameter. Hence, we can use time-series data on portfolio holdings to test whether the optimal portfolio weight is (primarily) U-shaped across states of the economy. A number of studies find that stock market participation is limited, especially in normal and bad states of the economy; see, e.g., Mankiw and Zeldes (1991) and VissingJørgensen (2002). Although our model cannot generate non-participation in the stock market, we note that the individual's willingness to invest in the stock market decreases strongly as loss aversion goes up.

Furthermore, a loss-averse individual with an endogenous reference level optimally implements a pronounced life-cycle investment strategy that reduces risk exposure with age, even without human capital; see Section 5.3. This finding holds true for a wide range of parameter values; see Section 5.3 and Figure 5. Furthermore, it holds true not only for the median scenario but also for other scenarios. We can test the hypothesis of a life-cycle investment strategy in the data. Although data seems to provide limited support for our hypothesis (see, e.g., the 2013 Survey of Consumer Finances), many financial advisors recommend a life-cycle investment strategy to their clients; see also Morningstar (2017) and the very recent evidence of substantial reduction of the risky share over the life-cycle in Fagereng, Gottlieb, and Guiso (2017).

\subsection{Probability Weighting}

He and Zhou (2016) show that probability weighting can generate an endogenous floor on wealth. Also, Carlson and Lazrak (2014) show that in a model with rank-dependent utility (RDU), probability weighting leads to a high degree of downward protection 31 Probability

\footnotetext{
${ }^{31}$ Carlson and Lazrak (2014) do not assume loss aversion nor endogenous updating of the reference level.
} 
weighting may therefore be another important ingredient of why individuals wish to protect against downside risk and hence do not want to participate in the stock market. Indeed, if individuals overweight the probability of tail events such as financial crises, then individuals may be reluctant to invest in risky stocks. We briefly indicate how we can incorporate probability weighting into our setting with intertemporal consumption and endogenous updating of the reference level, but leave a detailed analysis for future research. Instead of maximizing expected lifetime utility $U_{0}$ (see (1)), the individual now aims to maximize

$$
\int_{0}^{T} e^{-\delta t} V\left(c_{t}-\theta_{t}\right) \mathrm{d} t
$$

subject to the dynamic budget constraint, where

$$
\begin{aligned}
V\left(c_{t}-\theta_{t}\right)= & \int_{0}^{\infty} x^{\gamma_{G}} \mathrm{~d}\left[-w_{t, G}\left(1-\mathbb{P}\left[\left(c_{t}-\theta_{t}\right)^{+} \leq x\right]\right)\right] \\
& -\kappa \int_{0}^{\infty} x^{\gamma_{L}} \mathrm{~d}\left[-w_{t, L}\left(1-\mathbb{P}\left[\left(c_{t}-\theta_{t}\right)^{-} \leq x\right]\right)\right] .
\end{aligned}
$$

Here, the functions $w_{t, G}$ and $w_{t, L}$ transform the decumulative distributions functions of gains and losses, respectively. The individual's optimization problem can be solved in two steps. First, under technical conditions (see van Bilsen (2015), Chapter 3), we can use the quantile method (see, e.g., He and Zhou (2011)) to convert (20) into an ordinary expectation. Next, we can use the solution techniques of the current paper to obtain the optimal consumption and portfolio policies.

\section{Welfare Analysis}

This section considers a loss-averse individual with (initially) a constant reference level who delegates his consumption and portfolio decisions to a professional asset manager. The asset manager offers strategies that are exclusively based on conventional CRRA utility. They differ only in the degree of riskiness. The value of the CRRA parameter - which characterizes the CRRA utility function - determines the degree of riskiness of the strategies offered. The lossaverse individual chooses the value of the CRRA parameter such that the difference between his optimal utility (i.e., the utility associated with the optimal loss-averse strategy) and his actual utility (i.e., the utility associated with the CRRA strategy) be minimized. Because the individual's actual utility differs from the individual's optimal utility, the individual suffers a welfare loss. The welfare loss can be entirely attributed to the misspecification of the utility function. This section explores the size of this welfare loss; further details on the computation of the welfare losses can be found in Appendix F

Interestingly, they find that RDU investors invest more in the stock market as they become richer. This behavior is consistent with our model findings. 
Using the same parameter values as in Section 5.2 , we find that a loss-averse individual with a constant reference level who only has access to strategies based on CRRA utility suffers a welfare loss of at least $2.96 \%$. We measure the welfare loss in terms of the relative decline in certainty equivalent consumption 32 The minimum welfare loss of $2.96 \%$ is obtained when the asset manager implements the strategy of a CRRA individual with relative risk aversion equal to 14.5. We can view this number as a measure of the individual's relative risk aversion. The next subsections explore how a change in the individual's preference parameters affects the size of the welfare loss.

\subsection{Constant Reference Level}

Figure 6(a) and Figure 7|(a) illustrate the optimal consumption choice of a 65-year-old loss-averse individual for various values of the (constant) reference level $\theta$. As shown by these figures, the larger the reference level, the higher is the desired protection against low consumption levels, and the more upside potential is sacrificed. The dash-dotted lines correspond to a reference level of zero. The dash-dotted line in Figure $6($ a) would coincide with the consumption strategy of a CRRA individual with relative risk aversion equal to 0.6. The minimum welfare loss is thus zero by definition in this case; see also Table $3($ a), A loss-averse individual with a positive reference level always suffers a welfare loss, as shown by Table $3($ a).

\subsection{Loss Aversion Parameter}

Figure 6(b) and Figure 7](b) illustrate the optimal consumption choice of a 65-year-old lossaverse individual for various values of the loss aversion parameter $\kappa$. As illustrated by these figures, the probability of losses decreases as $\kappa$ increases. Intuitively, the more loss-averse the individual is, the more the portfolio strategy is aimed at avoiding losses. Indeed, as shown by Table $3(\mathrm{~b})$, the individual requests a more risk-averse strategy as the value of the loss aversion parameter $\kappa$ increases. Furthermore, the minimum welfare loss is a decreasing function of $\kappa$. Indeed, if the loss aversion parameter $\kappa$ is (very) high, then we can adequately approximate the optimal loss-averse strategy by the risk-free strategy (which is a special case of the CRRA strategy), especially in bad and normal states of the economy.

\subsection{Curvature Parameter for Gains}

Figure $6($ (c) and Figure $7(\mathrm{c})$ illustrate the optimal consumption choice of a 65-year-old loss-averse individual for various values of the curvature parameter for gains $\gamma_{G}$. The individual becomes less risk averse in the gain domain as $\gamma_{G}$ increases; see also the last column in Table 3 (c). That is, the larger the curvature parameter for gains $\gamma_{G}$, the higher is the degree of upside potential.

\footnotetext{
${ }^{32}$ The certainty equivalent of a risky consumption strategy is defined to be the constant consumption level that yields indifference to the risky consumption strategy.
} 
A higher degree of upside potential comes, however, at the expense of a larger probability of losses and less downward protection. Furthermore, the minimum welfare loss is an increasing function of $\gamma_{G}$.

\subsection{Curvature Parameter for Losses}

Figure $6(\mathrm{~d})$ and Figure $7(\mathrm{~d})$ illustrate the optimal consumption choice of a 65 -year-old lossaverse individual for various values of the curvature parameter for losses $\gamma_{L}$. In case of riskaverse behavior in the loss domain, the individual's degree of risk-aversion in the loss domain is increasing in $\gamma_{L}$, as shown by Figure $6(\mathrm{~d})$ and Table $3\left[(\mathrm{~d})\right.$. That is, the larger $\gamma_{L}$, the more consumption is protected against stock price shocks. In case of risk-loving behavior in the loss domain, the probability of a loss is decreasing in $\gamma_{L}$; see Figure $7(\mathrm{~d})$, As shown by Table $3(\mathrm{~d})$, the minimum welfare loss is largest for low values of $\gamma_{L}$. Furthermore, because the probability of a loss is relatively low, the minimum welfare loss is not very sensitive to a change in the curvature parameter for losses $\gamma_{L}$.

\subsection{Reference Level Dynamics}

Table 3(e) shows the impact of a change in the dynamics of the reference level on the minimum welfare loss. The welfare loss is likely to exceed $10 \%$ if the individual endogenously updates his reference level over time. In particular, if $\alpha=0.3$ and $\beta=0.27$, the minimum welfare loss equals $11.05 \% 33$ The results reveal that endogeneity of the reference level strongly amplifies the welfare loss that our individual would incur if he followed the least suboptimal strategy among the conventional CRRA optimal consumption and portfolio policies.

\section{[Insert Figure 6, Figure 7 and Table 3 near here]}

\section{Conclusion}

We have explored how loss aversion and endogenous updating of the individual's reference level jointly affect conventional wisdom on optimal drawdown and investment strategies. We have shown that the optimal policies under these pronounced empirical regularities differ fundamentally from predictions of the conventional consumption and investment model based on CRRA utility. Instead of decreasing his consumption level proportionally after a drop in wealth, a loss-averse individual with an endogenous reference level protects current consumption and postpones reductions in current consumption following a wealth drop. We

\footnotetext{
${ }^{33}$ We note that if we also adapt the loss aversion and curvature parameter values as in Section 5.3 such that the individual's median level of relative risk aversion at age 50 will remain the same compared to the case without endogenous updating (taking $\kappa=5, \gamma_{G}=0.2, \gamma_{L}=1.3, \alpha=0.3$ and $\beta=0.3$ ), the minimum welfare loss would equal $7.0 \%$.
} 
have shown that this typically occurs already with a constant reference level and amplifies with higher degrees of endogeneity. The incentive to delay cuts in consumption after a wealth drop depends on the individual's current wealth level: a medium-wealth individual has a stronger incentive to postpone cuts in current consumption than a low-wealth and, in particular, a high-wealth individual. Furthermore, the individual's optimal portfolio weight in the risky stock is not constant across states of the economy as is predicted by the conventional consumption and investment model, but rather depends on how close current and future expected consumption levels are to the reference level. The individual implements a conservative investment strategy in normal states and typically a more aggressive strategy in good and bad states. Endogeneity of the reference level increases overall risk-taking and makes the individual adopt a life-cycle investment strategy, which reduces risk exposure with age, even without taking human capital into account.

The significant welfare losses incurred by implementing the least suboptimal conventional consumption and portfolio policies highlight the importance of adequate individual drawdown and investment decisions over the life-cycle, and motivate scrutiny over the design of such financial plans. 


\section{A General Financial Market}

This appendix outlines the general financial market with multiple risky assets and a non-constant investment opportunity set that we assume in Appendix $\mathrm{B}$ and in the proof of Theorem 4.1

The uncertainty in the financial market is represented by a filtered probability space $(\Omega, \mathcal{F}, \mathbb{F}, \mathbb{P})$, on which is defined a standard $N$-dimensional Brownian motion $\left\{Z_{t}\right\}_{t \in[0, T]}$. The filtration $\mathbb{F}=\left\{\mathcal{F}_{t}\right\}_{t \in[0, T]}$ is the augmentation under $\mathbb{P}$ of the natural filtration generated by the standard Brownian motion $\left\{Z_{t}\right\}_{t \in[0, T]}$. Throughout, equalities and inequalities between random variables will be understood to hold $\mathbb{P}$-almost surely.

The financial market is complete and consists of a (locally) risk-free asset and $N$ risky stocks which are traded continuously on the finite horizon $[0, T]$. Let us denote by $B_{t}$ and $S_{t}$ the riskfree asset price and the vector of risky stock prices at adult age $t$, respectively. The risk-free asset price $B_{t}$ evolves as follows:

$$
\frac{\mathrm{d} B_{t}}{B_{t}}=r_{t} \mathrm{~d} t, \quad B_{0} \geq 0 \text { given, }
$$

where $r_{t}$ denotes the risk-free interest rate at adult age $t$. The vector of risky stock prices $S_{t}$ satisfies the following dynamic equation:

$$
\frac{\mathrm{d} S_{t}}{S_{t}}=\mu_{t} \mathrm{~d} t+\sigma_{t} \mathrm{~d} Z_{t}, \quad S_{0} \geq 0 \text { given, }
$$

where $\mu_{t}$ and $\sigma_{t}$ represent the vector of expected returns and the volatility matrix at adult age $t$, respectively, and $\mathrm{d} Z_{t}$ denotes the increment to the Brownian motion that drives the vector of stock returns.

We assume that the risk-free rate process $\left\{r_{t}\right\}_{t \in[0, T]}$, the mean rate of return process $\left\{\mu_{t}\right\}_{t \in[0, T]}$, and the volatility process $\left\{\sigma_{t}\right\}_{t \in[0, T]}$ are all $\mathcal{F}_{t}$-progressively measurable and uniformly bounded. In addition, we assume that for every $t(\epsilon>0)$,

$$
\vartheta^{\top} \sigma_{t} \sigma_{t}^{\top} \vartheta \geq \epsilon\|\vartheta\|^{2} \quad \text { for all } \vartheta \in \mathbb{R}^{N}
$$

with $T$ denoting the transpose sign. The strong non-degeneracy condition (24) implies that $\sigma_{t}$ is invertible for every $t$.

Let $M_{t}$ denote the stochastic discount factor at adult age $t$ which is defined as follows:

$$
M_{t}=\exp \left\{-\int_{0}^{t} r_{s} \mathrm{~d} s-\int_{0}^{t} \lambda_{s}^{\top} \mathrm{d} Z_{s}-\frac{1}{2} \int_{0}^{t} \sum_{i=1}^{N} \lambda_{i s}^{2} \mathrm{~d} s\right\} .
$$


Here, $\lambda_{t}=\left(\lambda_{1 t}, \lambda_{2 t}, \ldots, \lambda_{N t}\right)$ represents the vector of market prices of risk at adult age $t$ :

$$
\lambda_{t}=\sigma_{t}^{-1}\left(\mu_{t}-r_{t}\right)
$$

Let $\pi_{t}$ and $W_{t}$ denote the vector of total dollar amounts invested in the risky stocks and the individual's total wealth at adult age $t$, respectively. Total wealth $W_{t}$ satisfies the following dynamic budget constraint:

$$
\mathrm{d} W_{t}=\left(r_{t} W_{t}+\pi_{t}^{\top} \sigma_{t} \lambda_{t}-c_{t}\right) \mathrm{d} t+\pi_{t}^{\top} \sigma_{t} \mathrm{~d} Z_{t}, \quad W_{0} \geq 0 \text { given }
$$

The consumption process $\left\{c_{t}\right\}_{t \in[0, T]}$ and the portfolio process $\left\{\pi_{t}\right\}_{t \in[0, T]}$ are assumed to be $\mathcal{F}_{t^{-}}$ progressively measurable. We impose the following integrability conditions, which we assume to be satisfied for any consumption and portfolio process:

$$
\int_{0}^{T} \pi_{t}^{\top} \sigma_{t} \sigma_{t}^{\top} \pi_{t} \mathrm{~d} t<\infty, \quad \int_{0}^{T}\left|\pi_{t}\left(\mu_{t}-r_{t}\right)\right| \mathrm{d} t<\infty, \quad \mathbb{E}\left[\int_{0}^{T}\left|c_{t}\right|^{r} \mathrm{~d} t\right]<\infty \text { for all } r \in \mathbb{R} .
$$

The individual now faces the following optimization problem:

$$
\begin{array}{ll}
\max _{c_{t}, \pi_{t}: 0 \leq t \leq T} & \mathbb{E}\left[\int_{0}^{T} e^{-\delta t} v\left(c_{t}-\theta_{t}\right) \mathrm{d} t\right] \\
\text { s.t. } & \mathrm{d} W_{t}=\left(r W_{t}+\pi_{t}^{\top} \sigma \lambda-c_{t}\right) \mathrm{d} t+\pi_{t}^{\top} \sigma \mathrm{d} Z_{t}, \\
& \mathrm{~d} \theta_{t}=\left(\beta c_{t}-\alpha \theta_{t}\right) \mathrm{d} t, \\
& \theta_{t}-c_{t} \leq L_{t}^{\max }, \text { for all } t \in[0, T] .
\end{array}
$$

We note that in the general financial market of Appendix A with multiple risky assets and a non-constant investment opportunity set, the individual's static variational problem remains the same and is given by (11).

\section{B Dual Optimization Problem}

Schroder and Skiadas (2002) show that a generic consumption and portfolio choice model with linear internal habit formation can be mechanically transformed into a dual consumption and portfolio choice model without linear internal habit formation. Denote by $\widehat{c}_{t}$ the individual's dual (or surplus) consumption choice at adult age $t$; that is, $\widehat{c}_{t}=c_{t}-\theta_{t}$. The individual's 
optimization problem (11) is equivalent to the following dual optimization problem:

$$
\begin{array}{ll}
\max _{\widehat{c}_{t}: 0 \leq t \leq T} & \mathbb{E}\left[\int_{0}^{T} e^{-\delta t} v\left(\widehat{c}_{t}\right) \mathrm{d} t\right], \\
\text { s.t. } & \mathbb{E}\left[\int_{0}^{T} \frac{\widehat{M}_{t}}{\widehat{M}_{0}} \widehat{c}_{t} \mathrm{~d} t\right] \leq \widehat{W}_{0}, \\
& -\widehat{c}_{t} \leq L_{t}^{\max } \text { for all } t \in[0, T] .
\end{array}
$$

Here, $\widehat{M}_{t}$ and $\widehat{W}_{0}$ represent the dual counterparts of the stochastic discount factor $M_{t}$ and the individual's initial total wealth $W_{0}$, respectively.

The relationship between the optimization problem (11) and the dual optimization problem (30) is characterized in terms of the quantity $F_{t}$ :

$$
F_{t}=\frac{1}{M_{t}} \mathbb{E}_{t}\left[\int_{t}^{T} M_{s} e^{-(\alpha-\beta)(s-t)} \mathrm{d} s\right]
$$

We can view $F_{t}$ as the price of a bond paying a continuous coupon. The dual stochastic discount factor $\widehat{M}_{t}$ and the individual's dual initial total wealth $\widehat{W}_{0}$ are given by

$$
\begin{gathered}
\widehat{M}_{t}=M_{t}\left(1+\beta F_{t}\right), \\
\widehat{W}_{0}=\frac{W_{0}-F_{0} \theta_{0}}{1+\beta F_{0}} .
\end{gathered}
$$

Furthermore, the individual's dual reference level is equal to the individual's (primal) reference level; that is,

$$
\widehat{\theta}_{t}=e^{-(\alpha-\beta) t} \widehat{\theta}_{0}+\beta \int_{0}^{t} e^{-(\alpha-\beta)(t-s)} \widehat{c}_{s} \mathrm{~d} s=\theta_{t}
$$

Dual (or surplus) total wealth $\widehat{W}_{t}$ is defined as follows:

$$
\widehat{W}_{t}=\frac{1}{\widehat{M}_{t}} \mathbb{E}_{t}\left[\int_{t}^{T} \widehat{M}_{s} \widehat{c}_{s} \mathrm{~d} s\right]
$$

Dual total wealth $\widehat{W}_{t}$ is invested in a dual financial market which is characterized by the dual risk-free rate $\widehat{r}_{t}$, the dual volatility matrix $\widehat{\sigma}_{t}$, and the vector of dual market prices of risk $\widehat{\lambda}_{t}$ :

$$
\begin{gathered}
\widehat{r}_{t}=\beta+\frac{r_{t}-\alpha \beta F_{t}}{1+\beta F_{t}}, \\
\widehat{\sigma}_{t}=\sigma_{t}, \\
\widehat{\lambda}_{t}=\lambda_{t}-\frac{\beta}{1+\beta F_{t}} \int_{t}^{T} e^{-(\alpha-\beta)(s-t)} P_{t, s} \Psi_{t, s} \mathrm{~d} s .
\end{gathered}
$$


Here, $P_{t, s}$ corresponds to the price of a default-free unit discount bond that matures at adult age $s \geq t$ and $\Psi_{t, s}$ stands for the volatility of the instantaneous return on such a bond (all in the primal financial market).

Dual wealth $\widehat{W}_{t}$ is subject to the following dynamic equation:

$$
\mathrm{d} \widehat{W}_{t}=\left(\widehat{r}_{t} \widehat{W}_{t}+\widehat{\pi}_{t}^{\top} \widehat{\sigma}_{t} \widehat{\lambda}_{t}-\widehat{c}_{t}\right) \mathrm{d} t+\widehat{\pi}_{t}^{\top} \widehat{\sigma}_{t} \mathrm{~d} Z_{t}, \quad \widehat{W}_{0}=\frac{W_{0}-F_{0} \theta_{0}}{1+\beta F_{0}}
$$

Here, $\widehat{\pi}_{t}$ denotes the the dual portfolio choice.

The following proposition is adapted from Schroder and Skiadas (2002).

Proposition 1 (relationship Between dual solution And PRimal solution). Suppose that we have solved the dual optimization problem (30). Denote by $\widehat{c}_{t}^{*}$ the optimal dual consumption choice, by $\widehat{\theta}_{t}^{*}$ the optimal dual reference level, by $\widehat{W}_{t}^{*}$ the individual's optimal dual wealth, and by $\widehat{\pi}_{t}^{*}$ the optimal dual portfolio choice. Then:

- The individual's optimal consumption choice at adult age $t$ is given by

$$
c_{t}^{*}=\widehat{c}_{t}^{*}+\widehat{\theta}_{t}^{*} .
$$

- Optimal wealth at adult age $t$ is given by

$$
W_{t}^{*}=\widehat{W}_{t}^{*}+\beta F_{t} \widehat{W}_{t}^{*}+F_{t} \widehat{\theta}_{t}^{*}
$$

- The individual's optimal portfolio choice at adult age $t$ is given by

$$
\pi_{t}^{*}=\widehat{\pi}_{t}^{*}+\beta F_{t} \widehat{\pi}_{t}^{*}+\left(\beta \widehat{W}_{t}^{*}+\widehat{\theta}_{t}^{*}\right)\left(\widehat{\sigma}_{t}\right)^{-1} \int_{t}^{T} e^{-(\alpha-\beta)(s-t)} P_{t, s} \Psi_{t, s} \mathrm{~d} s
$$

Proposition 1 shows how to transform the optimal solution of the dual problem (30) back into the optimal solution of the individual's maximization problem 11.

\section{Proofs of Theorems}

First, we note that in the general financial market of Appendix A with multiple risky assets and a non-constant investment opportunity set, Theorem 4.1 applies identically.

Proof of Theorem 4.1: The proof uses some of the techniques developed by Basak and Shapiro (2001) and Berkelaar et al. (2004) in order to deal with pseudo-concavity and non-differentiability aspects of the individual's optimization problem. We adapt these techniques to our setting with intertemporal consumption. 
The dual optimization problem, which is equivalent to the optimization problem (11), is defined as follows:

$$
\begin{array}{ll}
\max _{\widehat{c_{t}}: 0 \leq t \leq T} & \mathbb{E}\left[\int_{0}^{T} e^{-\delta t} v\left(\widehat{c}_{t}\right) \mathrm{d} t\right], \\
\text { s.t. } & \mathbb{E}\left[\int_{0}^{T} \frac{\widehat{M}_{t}}{\widehat{M}_{0}} \widehat{c}_{t} \mathrm{~d} t\right] \leq \widehat{W}_{0}, \\
& -\widehat{c}_{t} \leq L_{t}^{\max } \quad \text { for all } t \in[0, T] .
\end{array}
$$

The corresponding Lagrangian $\mathcal{L}$ is given by

$$
\begin{aligned}
\mathcal{L} & =\mathbb{E}\left[\int_{0}^{T} e^{-\delta t} v\left(\widehat{c}_{t}\right) \mathrm{d} t\right]+y\left(\widehat{W}_{0}-\mathbb{E}\left[\int_{0}^{T} \frac{\widehat{M}_{t}}{\widehat{M}_{0}} \widehat{c}_{t} \mathrm{~d} t\right]\right) \\
& =\int_{0}^{T} \mathbb{E}\left[e^{-\delta t} v\left(\widehat{c}_{t}\right)-y \frac{\widehat{M}_{t}}{\widehat{M}_{0}} \widehat{c}_{t}\right] \mathrm{d} t+y \widehat{W}_{0} .
\end{aligned}
$$

Here, $y$ denotes the Lagrange multiplier associated with the static budget constraint in (40). The individual aims to maximize $e^{-\delta t} v\left(\widehat{c}_{t}\right)-y \widehat{M}_{t} \widehat{c}_{t} / \widehat{M}_{0}$ subject to $-\widehat{c}_{t} \leq L_{t}^{\max }$. Denote by $v_{G}$ the part of the utility function above the reference level, and by $v_{L}$ the part of the utility function below the reference level. Let $c_{t, G}^{*}$ and $c_{t, L}^{*}$ be the optimal solutions of the functions $v_{G}$ and $v_{L}$, respectively.

We first consider the case where the individual is risk averse in the loss domain. Due to the concavity of $v_{G}$ and $v_{L}$, the optimal dual consumption choices $c_{t, G}^{*}$ and $c_{t, L}^{*}$ satisfy the following optimality conditions (for $j=G, L)$

$$
\begin{gathered}
e^{-\delta t} v_{j}^{\prime}\left(c_{t, j}^{*}\right)=y \widehat{M}_{t} / \widehat{M}_{0}-x_{t, j}, \\
-c_{t, j}^{*} \leq L_{t}^{\max }, \\
x_{t, j}\left(c_{t, j}^{*}+L_{t}^{\max }\right)=0, \\
x_{t, j} \geq 0 .
\end{gathered}
$$

Here, $x_{t, j}(j=G, L)$ denotes the Lagrange multiplier associated with the constraint on dual consumption. After solving the optimality conditions, we obtain the following two local maxima:

$$
\begin{gathered}
c_{t, G}^{*}=\left(\frac{y e^{\delta t} \widehat{M}_{t}}{\gamma_{G} \widehat{M}_{0}}\right)^{\frac{1}{\gamma_{G}-1}}, \\
c_{t, L}^{*}=-\min \left\{\left(\frac{y e^{\delta t} \widehat{M}_{t}}{\kappa \gamma_{L} \widehat{M}_{0}}\right)^{\frac{1}{\gamma_{L}-1}}, L_{t}^{\max }\right\} .
\end{gathered}
$$

\footnotetext{
${ }^{34}$ We denote the derivative of a function $f$ at a point $a$ by $f^{\prime}(a)$.
} 
To determine the global maximum $\widehat{c}_{t}^{*}$, we introduce the following function:

$$
\begin{aligned}
f\left(\frac{\widehat{M}_{t}}{\widehat{M}_{0}}\right)= & e^{-\delta t} v\left(c_{t, G}^{*}\right)-y \frac{\widehat{M}_{t}}{\widehat{M}_{0}} c_{t, G}^{*}-\left(e^{-\delta t} v\left(c_{t, L}^{*}\right)-y \frac{\widehat{M}_{t}}{\widehat{M}_{0}} c_{t, L}^{*}\right) \\
= & e^{-\delta t}\left(1-\gamma_{G}\right)\left(\frac{y e^{\delta t} \widehat{M}_{t}}{\gamma_{G} \widehat{M}_{0}}\right)^{\frac{\gamma_{G}}{\gamma_{G}-1}}+e^{-\delta t} \kappa \min \left\{\left(\frac{y e^{\delta t} \widehat{M}_{t}}{\kappa \gamma_{L} \widehat{M}_{0}}\right)^{\frac{1}{\gamma_{L}-1}}, L_{t}^{\max }\right\}^{\gamma_{L}} \\
& -y \frac{\widehat{M}_{t}}{\widehat{M}_{0}} \min \left\{\left(\frac{y e^{\delta t} \widehat{M}_{t}}{\kappa \gamma_{L} \widehat{M}_{0}}\right)^{\frac{1}{\gamma_{L}-1}}, L_{t}^{\max }\right\} .
\end{aligned}
$$

The global maximum $\widehat{c}_{t}^{*}$ is equal to $c_{t, G}^{*}$ if $f\left(\frac{\widehat{M}_{t}}{\widehat{M}_{0}}\right) \geq 0$; and equals $c_{t, L}^{*}$ otherwise. It follows that $\lim _{\frac{\widehat{M}_{t}}{\widehat{M}_{0} \rightarrow \infty}} f\left(\frac{\widehat{M}_{t}}{\widehat{M}_{0}}\right)=-\infty, \lim _{\frac{\widehat{M}_{t}}{\widehat{M}_{0}} \rightarrow 0} f\left(\frac{\widehat{M}_{t}}{\widehat{M}_{0}}\right)=\infty$ and $f^{\prime}\left(\frac{\widehat{M}_{t}}{\widehat{M}_{0}}\right)<0$ for all $\frac{\widehat{M}_{t}}{\widehat{M}_{0}}$. Hence, $f\left(\frac{\widehat{M}_{t}}{\widehat{M}_{0}}\right)$ is strictly decreasing. As a result, $f\left(\frac{\widehat{M}_{t}}{\widehat{M}_{0}}\right)$ has one zero in the interval $(0, \infty)$. We define $\xi_{t}$ such that $f\left(\xi_{t}\right)=0$. The global maximum $\widehat{c}_{t}^{*}$ is equal to $c_{t, G}^{*}$ if $\frac{\widehat{M}_{t}}{\widehat{M}_{0}} \leq \xi_{t}$; and equals $c_{t, L}^{*}$ otherwise.

We now consider the case where the individual is risk loving in the loss domain. Due to the concavity of $v_{G}$, the optimal dual consumption choice $c_{t, G}^{*}$ satisfies the following optimality conditions:

$$
\begin{gathered}
e^{-\delta t} v_{G}^{\prime}\left(c_{t, G}^{*}\right)=y \widehat{M}_{t} / \widehat{M}_{0}-x_{t, G}, \\
-c_{t, G}^{*} \leq L_{t}^{\max }, \\
x_{t, G}\left(c_{t, G}^{*}+L_{t}^{\max }\right)=0 \\
x_{t, G} \geq 0 .
\end{gathered}
$$

After solving the optimality conditions, we obtain the following local maximum:

$$
c_{t, G}^{*}=\left(\frac{y e^{\delta t} \widehat{M}_{t}}{\gamma_{G} \widehat{M}_{0}}\right)^{\frac{1}{\gamma_{G}^{-1}}} .
$$

Due to the convexity of $v_{L}$, the optimal dual consumption choice $c_{t, L}^{*}$ lies at a corner point of the feasible region. Hence, the only two possible candidates for $c_{t, L}^{*}$ are $-L_{t}^{\max }$ and 0 .

To determine the global maximum $\widehat{c}_{t}^{*}$, we introduce the following function:

$$
g\left(\frac{\widehat{M}_{t}}{\widehat{M}_{0}}\right)=e^{-\delta t} v\left(c_{t, G}^{*}\right)-y \frac{\widehat{M}_{t}}{\widehat{M}_{0}} c_{t, G}^{*}-\left(e^{-\delta t} v\left(c_{t, L}^{*}\right)-y \frac{\widehat{M}_{t}}{\widehat{M}_{0}} c_{t, L}^{*}\right) .
$$


The global maximum $\widehat{c}_{t}^{*}$ is equal to $c_{t, G}^{*}$ if $g\left(\frac{\widehat{M}_{t}}{\widehat{M}_{0}}\right) \geq 0$; and equals $c_{t, L}^{*}$ otherwise. We distinguish between the following two cases:

- $c_{t, L}^{*}=0$. Straightforward computations show that $g\left(\frac{\widehat{M}_{t}}{\widehat{M}_{0}}\right)$ is given by

$$
g\left(\frac{\widehat{M}_{t}}{\widehat{M}_{0}}\right)=e^{-\delta t}\left(1-\gamma_{G}\right)\left(\frac{y e^{\delta t} \widehat{M}_{t}}{\gamma_{G} \widehat{M}_{0}}\right)^{\frac{\gamma_{G}}{\gamma_{G}-1}}
$$

Since $0<\gamma_{G}<1$ and $y>0$, it follows that $g\left(\frac{\widehat{M}_{t}}{\widehat{M}_{0}}\right)>0$ for all $\frac{\widehat{M}_{t}}{\widehat{M}_{0}}$. We conclude that $c_{t, L}^{*}=0$ is never optimal.

- $c_{t, L}^{*}=-L_{t}^{\max }$. Straightforward computations show that $g\left(\frac{\widehat{M}_{t}}{\widehat{M}_{0}}\right)$ is given by

$$
g\left(\frac{\widehat{M}_{t}}{\widehat{M}_{0}}\right)=e^{-\delta t}\left(1-\gamma_{G}\right)\left(\frac{y e^{\delta t} \widehat{M}_{t}}{\gamma_{G} \widehat{M}_{0}}\right)^{\frac{\gamma_{G}}{\gamma_{G}-1}}+e^{-\delta t} \kappa\left(L_{t}^{\max }\right)^{\gamma_{L}}-y \frac{\widehat{M}_{t}}{\widehat{M}_{0}} L_{t}^{\max }
$$

It follows that $g\left(\frac{\widehat{M}_{t}}{\widehat{M}_{0}}\right)>0$ for all $\frac{\widehat{M}_{t}}{\widehat{M}_{0}} \leq \frac{\kappa}{y} e^{-\delta t}\left(L_{t}^{\max }\right)^{\gamma_{L}-1}$. Also, $\lim _{\widehat{M}_{t} \rightarrow \infty} g\left(\frac{\widehat{M}_{t}}{\widehat{M}_{0}}\right)=-\infty$ and $g^{\prime}\left(\frac{\widehat{M}_{t}}{\widehat{M}_{0}}\right)<0$ for all $\frac{\widehat{M}_{t}}{\widehat{M}_{0}}$. Hence, $g\left(\frac{\widehat{M}_{t}}{\widehat{M}_{0}}\right)$ is strictly decreasing. As a result, $g\left(\frac{\widehat{M}_{t}}{\widehat{M}_{0}}\right)$ has one zero in the interval $\left(\frac{\kappa}{y} e^{-\delta t}\left(L_{t}^{\max }\right)^{\gamma_{L}-1}, \infty\right)$. We define $\zeta_{t}$ such that $g\left(\zeta_{t}\right)=0$. It follows that the global maximum $\widehat{c}_{t}^{*}$ is equal to $c_{t, G}^{*}$ if $\frac{\widehat{M}_{t}}{\widehat{M}_{0}} \leq \zeta_{t}$; and equals $c_{t, L}^{*}$ otherwise. A standard verification (see, e.g., Karatzas and Shreve (1998), p. 103) that the optimal solutions obtained from the Lagrangian are the optimal solutions of the dual problem completes the proof. Q.E.D.

Proof of Theorem 4.2: We first compute optimal dual total wealth, which is defined as follows:

$$
\widehat{W}_{t}^{*}=\frac{1}{\widehat{M}_{t}} \mathbb{E}_{t}\left[\int_{t}^{T} \widehat{M}_{s} \widehat{c}_{s}^{*} \mathrm{~d} s\right]
$$

Suppose that the individual is risk averse in the loss domain. Substitution of the optimal dual consumption choice $\widehat{c}_{s}^{*}$ into equation (56) yields

$$
\widehat{W}_{t}^{*}=\widehat{W}_{t}^{G *}-\widehat{W}_{t}^{L *}
$$


where

$$
\begin{aligned}
& \left.\widehat{W}_{t}^{G *}=\frac{1}{\widehat{M}_{t}} \mathbb{E}_{t}\left[\int_{t}^{T} \widehat{M}_{s}\left(\frac{y e^{\delta s} \widehat{M}_{s}}{\gamma_{G} \widehat{M}_{0}}\right)^{\frac{1}{\gamma_{G}^{-1}}} \mathbb{1}_{\left[\frac{\widehat{M}_{s}}{\widehat{M}_{0}} \leq \xi_{s}\right.}\right] \mathrm{d} s\right] \\
& =\left(\frac{y e^{\delta t} \widehat{M}_{t}}{\gamma_{G} \widehat{M}_{0}}\right)^{\frac{1}{\gamma_{G}-1}} \mathbb{E}_{t}\left[\int_{t}^{T}\left(\frac{\widehat{M}_{s}}{\widehat{M}_{t}}\right)^{\frac{\gamma_{G}}{\gamma_{G}-1}} e^{\frac{\delta(s-t)}{\gamma_{G}^{-1}}} \mathbb{1}_{\left[\widehat{M}_{s} \leq \xi_{s}\right.} \mathrm{d} s\right] \text {, } \\
& \widehat{W}_{t}^{L *}=\frac{1}{\widehat{M}_{t}} \mathbb{E}_{t}\left[\int_{t}^{T} \widehat{M}_{s}\left\{\left(\frac{y e^{\delta s} \widehat{M}_{s}}{\kappa \gamma_{L} \widehat{M}_{0}}\right)^{\frac{1}{\gamma_{L}-1}} \mathbb{1}_{\left[\xi_{s}<\widehat{M}_{s}\right.}<\max \left\{\xi_{s}, \eta_{s}\right\}\right]\right. \\
& \left.\left.\left.+L_{s}^{\max } \mathbb{1}_{\left[\frac{\widehat{M}_{s}}{\widehat{M}_{0}} \geq \max \left\{\xi_{s}, \eta_{s}\right\}\right.}\right] \mathrm{d} s\right\}\right] \\
& =\left(\frac{y e^{\delta t} \widehat{M}_{t}}{\kappa \gamma_{L} \widehat{M}_{0}}\right)^{\frac{1}{\gamma_{L}-1}} \mathbb{E}_{t}\left[\int_{t}^{T}\left(\frac{\widehat{M}_{s}}{\widehat{M}_{t}}\right)^{\frac{\gamma_{L}}{\gamma_{L}-1}} e^{\frac{\delta(s-t)}{\gamma_{L}-1}} \mathbb{1}_{\left[\xi_{s}<\frac{\widehat{M}_{s}}{\widehat{M}_{0}}<\max \left\{\xi_{s}, \eta_{s}\right\}\right.}\right] \\
& \left.+\mathbb{E}_{t}\left[\int_{t}^{T} \frac{\widehat{M}_{s}}{\widehat{M}_{t}} L_{s}^{\max } \mathbb{1}_{\left[\widehat{M}_{s}\right.} \geq \max \left\{\xi_{s}, \eta_{s}\right\}\right] \mathrm{d} s\right] .
\end{aligned}
$$

Here, $\eta_{t}=\frac{\kappa \gamma_{L}}{y e^{\delta t}}\left(L_{t}^{\max }\right)^{\gamma_{L}-1}$. A closed-form expression for $\widehat{W}_{t}^{*}$ can be computed in case the investment opportunity set is constant. We find

$$
\begin{aligned}
&\left.\mathbb{E}_{t}\left[\frac{\widehat{M}_{s}}{\widehat{M}_{t}} L_{s}^{\max } \mathbb{1}_{\left[\frac{\widehat{M}_{s}}{\widehat{M}_{0}} \geq \max \left\{\xi_{s}, \eta_{s}\right\}\right.}\right]\right]=e^{-\int_{t}^{s} \widehat{r}_{u} \mathrm{~d} u} L_{s}^{\max } \mathcal{N}\left[-d_{1}\left(\max \left\{\xi_{s}, \eta_{s}\right\}\right)\right], \\
&\left.\mathbb{E}_{t}\left[\left(\frac{\widehat{M}_{s}}{\widehat{M}_{t}}\right)^{\frac{\gamma_{G}}{\gamma_{G}-1}} e^{\frac{\delta(s-t)}{\gamma_{G}-1}} \mathbb{1}_{\left[\frac{\widehat{M}_{s}}{\bar{M}_{0}} \leq \xi_{s}\right]}\right]\right]=e^{-\int_{t}^{s} \Gamma_{u} \mathrm{~d} u} \mathcal{N}\left[d_{2}\left(\xi_{s}\right)\right], \\
&\left.\mathbb{E}_{t}\left[\left(\frac{\widehat{M}_{s}}{\widehat{M}_{t}}\right)^{\frac{\gamma_{L}}{\gamma_{L}-1}} e^{\frac{\delta(s-t)}{\gamma_{L}-1}} \mathbb{1}_{\left[\xi_{s}<\widehat{M}_{s}\right.}<\max \left\{\xi_{s}, \eta_{s}\right\}\right]\right]=e^{-\int_{t}^{s} \Pi_{u} \mathrm{~d} u} \\
& \times\left(\mathcal{N}\left[d_{3}\left(\max \left\{\xi_{s}, \eta_{s}\right\}\right)\right]-\mathcal{N}\left[d_{3}\left(\xi_{s}\right)\right]\right) .
\end{aligned}
$$

Here, $\mathcal{N}$ is the cumulative distribution function of a standard normal random variable, and $\Gamma_{t}$, 
$\Pi_{t}, d_{1}(x), d_{2}(x)$ and $d_{3}(x)$ are defined as follows:

$$
\begin{gathered}
\Gamma_{t}=\frac{\delta-\gamma_{G} \widehat{r}_{t}}{1-\gamma_{G}}-\frac{1}{2} \frac{\gamma_{G}}{\left(1-\gamma_{G}\right)^{2}}\|\lambda\|^{2}, \\
\Pi_{t}=\frac{\delta-\gamma_{L} \widehat{r}_{u}}{1-\gamma_{L}}-\frac{1}{2} \frac{\gamma_{L}}{\left(1-\gamma_{L}\right)^{2}}\|\lambda\|^{2}, \\
d_{1}(x)=\frac{1}{\|\lambda\| \sqrt{s-t}} \cdot\left[\log (x)-\log \left(\frac{\widehat{M}_{t}}{\widehat{M}_{0}}\right)+\int_{t}^{s} \widehat{r}_{u} \mathrm{~d} u-\frac{1}{2}\|\lambda\|^{2}(s-t)\right], \\
d_{2}(x)=d_{1}(x)+\frac{\|\lambda\|}{1-\gamma_{G}} \sqrt{s-t}, \\
d_{3}(x)=d_{1}(x)+\frac{\|\lambda\|}{1-\gamma_{L}} \sqrt{s-t} .
\end{gathered}
$$

Substitution of the conditional expectations (60), 61) and (62) into equation (57) yields the optimal dual wealth.

Suppose now that the individual is risk loving in the loss domain. Substitution of the optimal dual consumption choice $\widehat{c}_{s}^{*}$ into equation (56) yields

$$
\widehat{W}_{t}^{*}=\widehat{W}_{t}^{G *}-\widehat{W}_{t}^{L *}
$$

where

$$
\begin{aligned}
& \widehat{W}_{t}^{G *}=\frac{1}{\widehat{M}_{t}} \mathbb{E}_{t}\left[\int_{t}^{T} \widehat{M}_{s}\left(\frac{y e^{\delta s} \widehat{M}_{s}}{\gamma_{G} \widehat{M}_{0}}\right)^{\frac{1}{\gamma_{G}^{-1}}} \mathbb{1}_{\left[\frac{\widehat{M}_{s}}{\widehat{M}_{0}} \leq \zeta_{s}\right]} \mathrm{d} s\right] \\
& =\left(\frac{y e^{\delta t} \widehat{M}_{t}}{\gamma_{G} \widehat{M}_{0}}\right)^{\frac{1}{\gamma_{G}{ }^{-1}}} \mathbb{E}_{t}\left[\int_{t}^{T}\left(\frac{\widehat{M}_{s}}{\widehat{M}_{t}}\right)^{\frac{\gamma_{G}}{\gamma_{G}-1}} e^{\frac{\delta(s-t)}{\gamma_{G}-1}} \mathbb{1}_{\left[\widehat{M}_{s} \leq \zeta_{s}\right.} \mathrm{d} s\right] \text {, } \\
& \widehat{W}_{t}^{L *}=\mathbb{E}_{t}\left[\int_{t}^{T} \frac{\widehat{M}_{s}}{\widehat{M}_{t}} L_{s}^{\max } \mathbb{1}_{\left[\frac{\widehat{M}_{s}}{\widehat{M}_{0}}>\zeta_{s}\right.} \mathrm{d} s\right] .
\end{aligned}
$$

A closed-form expression for $\widehat{W}_{t}^{*}$ can be computed in case the investment opportunity set is constant. We find

$$
\begin{gathered}
\left.\mathbb{E}_{t}\left[\frac{\widehat{M}_{s}}{\widehat{M}_{t}} L_{s}^{\max } \mathbb{1}_{\left[\widehat{M}_{s}\right.} \zeta_{\widehat{M}_{0}}\right]\right]=e^{-\int_{t}^{s} \widehat{r}_{u} \mathrm{~d} u} L_{s}^{\max } \mathcal{N}\left[-d_{1}\left(\zeta_{s}\right)\right], \\
\mathbb{E}_{t}\left[\left(\frac{\widehat{M}_{s}}{\widehat{M}_{t}}\right)^{\frac{\gamma_{G}}{\gamma_{G}-1}} e^{\frac{\delta(s-t)}{\gamma_{G}^{-1}}} \mathbb{1}_{\left[\frac{\widehat{M}_{s}}{\widehat{M}_{0}} \leq \zeta_{s}\right]}\right]=e^{-\int_{t}^{s} \Gamma_{u} \mathrm{~d} u} \mathcal{N}\left[d_{2}\left(\zeta_{s}\right)\right] .
\end{gathered}
$$

Substitution of 771 and 72 into 68 yields the optimal dual wealth. 
The optimal dual portfolio choice $\widehat{\pi}_{t}^{*}$ can be constructed using standard hedging arguments. We explicitly determine the optimal dual portfolio choice for the case of a constant investment opportunity set. To this end, it is convenient to express $\widehat{W}_{t}^{*}$ as a function of $t$ and the dual stochastic discount factor $\widehat{M}_{t}$; that is, $\widehat{W}_{t}^{*}=h\left(t, \widehat{M}_{t}\right)$ for some (regular) function $h$. Straightforward application of Itô's Lemma to the function $h$ yields

$$
\mathrm{d} \widehat{W}_{t}^{*}=\left[\frac{\partial h}{\partial t}-\frac{\partial h}{\partial \widehat{M}_{t}} \widehat{M}_{t} \widehat{r}_{t}+\frac{1}{2} \frac{\partial^{2} h}{\partial \widehat{M}_{t}^{2}} \widehat{M}_{t}^{2}\|\lambda\|^{2}\right] \mathrm{d} t-\frac{\partial h}{\partial \widehat{M}_{t}} \widehat{M}_{t} \lambda^{\top} \mathrm{d} Z_{t} .
$$

Comparing the diffusion part of the dynamic budget constraint (39) with the diffusion part of equation (73), we arrive at the dual optimal portfolio choice:

$$
\widehat{\pi}_{t}^{*}=-\frac{\partial h}{\partial \widehat{M}_{t}} \widehat{M}_{t} \lambda^{\top} \sigma^{-1}
$$

The individual's optimal (primal) portfolio choice follows from Schroder and Skiadas (2002):

$$
\pi_{t}^{*}=\widehat{\pi}_{t}^{*}+\beta F_{t} \widehat{\pi}_{t}^{*}
$$

We find:

- If the individual is risk averse in the loss domain, the optimal dual portfolio strategy is given by

$$
\widehat{\pi}_{t}^{*}=A_{t} \cdot \widehat{W}_{t}^{G *}+B_{t} \cdot \widehat{W}_{t}^{L *}
$$

where

$$
\begin{aligned}
& A_{t}=\frac{\sigma^{-1} \lambda}{\left(1-\gamma_{G}\right)}+\sigma^{-1} \lambda\left(\frac{y e^{\delta t} \widehat{M}_{t}}{\gamma_{G} \widehat{M}_{0}}\right)^{\frac{1}{\gamma_{G}-1}} \int_{t}^{T} e^{-\int_{t}^{s} \Gamma_{u} \mathrm{~d} u} \frac{\phi\left[d_{2}\left(\xi_{s}\right)\right]}{\|\lambda\| \sqrt{s-t}} \mathrm{~d} s\left(\widehat{W}_{t}^{G *}\right)^{-1}, \\
& B_{t}=-\left(\widehat{W}_{t}^{L *}\right)^{-1}\left(\frac{y e^{\delta t} \widehat{M}_{t}}{\kappa \gamma_{L} \widehat{M}_{0}}\right)^{\frac{1}{\gamma_{L}-1}} \\
& \left\{\int_{t}^{T} e^{-\int_{t}^{s} \Pi_{u} \mathrm{~d} u} \frac{\phi\left[d_{2}\left(\max \left\{\eta_{s}, \xi_{s}\right\}\right)\right]-\phi\left[d_{2}\left(\xi_{s}\right)\right]}{\|\lambda\| \sqrt{s-t}} \mathrm{~d} s\right. \\
& \left.+\frac{\sigma^{-1} \lambda}{\left(1-\gamma_{L}\right)} \int_{t}^{T} e^{-\int_{t}^{s} \Pi_{u} \mathrm{~d} u}\left(\mathcal{N}\left[d_{3}\left(\max \left\{\eta_{s}, \xi_{s}\right\}\right)\right]-\mathcal{N}\left[d_{3}\left(\xi_{s}\right)\right]\right) \mathrm{d} s\right\} \\
& +\left(\widehat{W}_{t}^{L *}\right)^{-1} \int_{t}^{T} e^{-\int_{t}^{s} \widehat{r}_{u} \mathrm{~d} u} L_{s}^{\max } \frac{\phi\left[-d_{1}\left(\max \left\{\eta_{s}, \xi_{s}\right\}\right)\right]}{\|\lambda\| \sqrt{s-t}} \mathrm{~d} s .
\end{aligned}
$$

- If the individual is risk loving in the loss domain, the optimal dual portfolio strategy is given by

$$
\widehat{\pi}_{t}^{*}=C_{t} \cdot \widehat{W}_{t}^{G *}+D_{t} \cdot \widehat{W}_{t}^{L *}
$$


where

$$
\begin{gathered}
C_{t}=\frac{\sigma^{-1} \lambda}{\left(1-\gamma_{G}\right)}+\sigma^{-1} \lambda\left(\frac{y e^{\delta t} \widehat{M}_{t}}{\gamma_{G} \widehat{M}_{0}}\right)^{\frac{1}{\gamma_{G}^{-1}}} \int_{t}^{T} e^{-\int_{t}^{s} \Gamma_{u} \mathrm{~d} u} \frac{\phi\left[d_{2}\left(\zeta_{s}\right)\right]}{\|\lambda\| \sqrt{s-t}} \mathrm{~d} s\left(\widehat{W}_{t}^{G *}\right)^{-1} \\
D_{t}=\sigma^{-1} \lambda \int_{t}^{T} e^{-\int_{t}^{s} \widehat{r}_{u} \mathrm{~d} u} L_{s}^{\max } \frac{\phi\left[-d_{1}\left(\zeta_{s}\right)\right]}{\|\lambda\| \sqrt{s-t}} \mathrm{~d} s\left(\widehat{W}_{t}^{L *}\right)^{-1}
\end{gathered}
$$

\section{Reference Level as an Average of Past Consumption Choices}

Fix $t$. We can write the individual's reference level (5) at adult age $t$ as follows:

$$
\theta_{t}=e^{-\alpha t} \theta_{0}+\int_{0}^{t} \omega_{s} c_{s} \mathrm{~d} s
$$

where

$$
\omega_{s}=\beta e^{-\alpha(t-s)} .
$$

The reference level is given by a weighted average of past consumption choices if the weights sum to unity; that is, if $e^{-\alpha t}+\int_{0}^{t} \omega_{s} \mathrm{~d} s=1$. Straightforward computations show that

$$
e^{-\alpha t}+\int_{0}^{t} \omega_{s} \mathrm{~d} s=e^{-\alpha t}+\int_{0}^{t} \beta e^{-\alpha(t-s)} \mathrm{d} s=e^{-\alpha t}+\beta \frac{1}{\alpha}\left(1-e^{-\alpha t}\right)
$$

It follows from 82 that if $\beta=\alpha$, the reference level is given by a weighted average of past consumption choices 35

\section{E Impact of a Current Shock on Future Consumption}

This appendix shows that the impact of a current shock on future median consumption is typically larger the further into the future consumption occurs (in other words, the individual has a strong incentive to postpone cuts in current consumption). For ease of exposition, we restrict ourselves to the case of infinite loss aversion (i.e., $\kappa=\infty$ ) and constant investment opportunities, so that consumption always stays above the reference level. Optimal median

\footnotetext{
${ }^{35}$ We note that in a discrete-time setting, $\beta$ should be taken slightly smaller than $\alpha$. More specifically, if

$$
\beta=\frac{1-e^{-\alpha \Delta t}}{\Delta t}
$$

then the reference level is given by a discrete-time weighted average of past consumption choices (here, $\Delta t$ denotes the time step).
} 
consumption at time $s>t$ is given by

$$
\begin{aligned}
\mathbb{M}_{t}\left[c_{s}^{*}\right] & =\mathbb{M}_{t}\left[\theta_{s}^{*}+\left(\frac{y e^{\delta s}}{\gamma_{G}}\right)^{\frac{1}{\gamma_{G}-1}}\left(\frac{\widehat{M}_{s}}{\widehat{M}_{0}}\right)^{\frac{1}{\gamma_{G}}}\right] \\
& =\mathbb{M}_{t}\left[e^{-\alpha s} \theta_{0}+\beta \int_{0}^{s} e^{-(\alpha-\beta)(s-v) \widehat{c}_{v}^{*}} \mathrm{~d} v+\left(\frac{y e^{\delta s}}{\gamma_{G}}\right)^{\frac{1}{\gamma_{G}-1}}\left(\frac{\widehat{M}_{s}}{\widehat{M}_{0}}\right)^{\frac{1}{\gamma_{G}-1}}\right] \\
& =\mathbb{M}_{t}\left[e^{-\alpha s} \theta_{0}+\beta \int_{0}^{s} e^{-(\alpha-\beta)(s-v)}\left(\frac{y e^{\delta v}}{\gamma_{G}}\right)^{\frac{1}{\gamma_{G}-1}}\left(\frac{\widehat{M}_{v}}{\widehat{M}_{0}}\right)^{\frac{1}{\gamma_{G}^{-1}}} \mathrm{~d} v\right. \\
& \left.+\left(\frac{y e^{\delta s}}{\gamma_{G}}\right)^{\frac{1}{\gamma_{G}-1}}\left(\frac{\widehat{M}_{s}}{\widehat{M}_{0}}\right)^{\frac{1}{\gamma_{G}-1}}\right] .
\end{aligned}
$$

Here, we have used the definitions of $\theta_{s}^{*}$ and $\widehat{c}_{v}^{*}$ (see (34) and (46)). Substituting the analytical expression of $\widehat{M}_{v}$ into 84 and computing the median, we arrive at

$$
\begin{aligned}
\mathbb{M}_{t}\left[c_{s}^{*}\right] & =\beta \int_{0}^{s} e^{-(\alpha-\beta)(s-v)}\left(\frac{y e^{\delta v}}{\gamma_{G}}\right)^{\frac{1}{\gamma_{G}-1}}\left(e^{\frac{1}{1-\gamma_{G}} \int_{0}^{v} \widehat{r}_{u} \mathrm{~d} u-\frac{1}{2} \frac{\lambda^{2} v}{\gamma_{G}-1}-\frac{\lambda}{\gamma_{G}} \int_{0}^{\min (v, t)} \mathrm{d} W_{u}}\right) \mathrm{d} v \\
& +e^{-\alpha s} \theta_{0}+\left(\frac{y e^{\delta s}}{\gamma_{G}}\right)^{\frac{1}{\gamma_{G}-1}}\left(e^{\frac{1}{1-\gamma_{G}} \int_{0}^{s} \widehat{r}_{u} \mathrm{~d} u-\frac{1}{2} \frac{\lambda^{2} s}{\gamma_{G}-1}-\frac{\lambda}{\gamma_{G}-1} \int_{0}^{t} \mathrm{~d} W_{u}}\right) .
\end{aligned}
$$

Note that the Brownian increments on the right-hand side of 85 have been realized and are thus non-random. Next, we compute $\partial \mathbb{M}_{t}\left[c_{s}^{*}\right] / \partial W_{t}$. We find

$$
\begin{aligned}
\frac{\partial \mathbb{M}_{t}\left[c_{s}^{*}\right]}{\partial W_{t}} & =\beta \int_{t}^{s} e^{-(\alpha-\beta)(s-v)} \frac{\lambda}{1-\gamma_{G}}\left(\frac{y e^{\delta v}}{\gamma_{G}}\right)^{\frac{1}{\gamma_{G}-1}} \mathbb{M}_{t}\left[\left(\frac{\widehat{M}_{v}}{\widehat{M}_{0}}\right)^{\frac{1}{\gamma_{G}-1}}\right] \mathrm{d} v \\
& +\frac{\lambda}{1-\gamma_{G}}\left(\frac{y e^{\delta s}}{\gamma_{G}}\right)^{\frac{1}{\gamma_{G}^{-1}}} \mathbb{M}_{t}\left[\left(\frac{\widehat{M}_{s}}{\widehat{M}_{0}}\right)^{\frac{1}{\gamma_{G}-1}}\right] .
\end{aligned}
$$

It follows from (86) that $\partial \mathbb{M}_{t}\left[c_{s}^{*}\right] / \partial W_{t}$ tends to increase as the horizon $s-t$ widens. Indeed, as $s$ increases, the interval over which the positive function

$$
f(v):=\beta e^{-(\alpha-\beta)(s-v)} \frac{\lambda}{1-\gamma_{G}}\left(\frac{y e^{\delta v}}{\gamma_{G}}\right)^{\frac{1}{\gamma_{G}-1}} \mathbb{M}_{t}\left[\left(\frac{\widehat{M}_{v}}{\widehat{M}_{0}}\right)^{\frac{1}{\gamma_{G}-1}}\right]
$$

is integrated increases. Furthermore, if $\delta$ is not too large, the second term on the right-hand side of 86 becomes larger as $s$ goes up. Hence, the individual has a preference to postpone adjustments in current consumption. 


\section{F Computation of Minimum Welfare Loss}

This appendix describes a numerical procedure for computing minimum welfare losses. This procedure is based on the assumptions that the investment opportunity set is constant and the individual can only invest in one risky stock. We introduce the following notation:

- $\gamma \in\{0,0.1, \ldots, 99.9,100\}$ : CRRA parameter (i.e., relative risk aversion coefficient of an individual with power utility);

- $\mathcal{S}$ : total number of simulations;

- $\Delta t$ : time step;

- $t_{n}=n \Delta t$ for $n=0, \ldots,\left\lfloor\frac{T}{\Delta t}\right\rfloor$.

The floor operator $\lfloor\cdot\rfloor$ rounds a number downward to its nearest integer.

To compute the minimum welfare loss associated with the CRRA consumption strategy, we apply the following steps:

1. We generate $\mathcal{S}$ trajectories of the stochastic discount factor $(s=1, \ldots, \mathcal{S})$ :

$$
M_{s, t_{n+1}}=M_{s, t_{n}}-r M_{s, t_{n}} \Delta t-\lambda M_{s, t_{n}} \sqrt{\Delta t} \epsilon_{s, t_{n}}, \quad n=0, \ldots,\left\lfloor\frac{T}{\Delta t}\right\rfloor .
$$

Here, $\epsilon_{s, t_{n}}$ is a standard normally distributed random variable.

2. We compute the optimal dual consumption choice $\widehat{c}_{s, t_{n}}^{*}$ for $s=1, \ldots, \mathcal{S}$ and $n=0, \ldots,\left\lfloor\frac{T}{\Delta t}\right\rfloor$. We note that the optimal dual consumption choice $\widehat{c}_{s, t_{n}}^{*}$ is a function of the dual stochastic discount factor $\widehat{M}_{s, t_{n}}=M_{s, t_{n}}\left(1+\beta F_{t_{n}}\right)$. Expected lifetime utility can now be approximated by

$$
\mathbb{E}\left[\int_{0}^{T} e^{-\delta t} v\left(\widehat{c}_{t}^{*}\right) \mathrm{d} t\right] \approx \frac{1}{\mathcal{S}} \sum_{s=1}^{\mathcal{S}} \sum_{n=0}^{\left\lfloor\frac{T}{\Delta t}\right\rfloor} e^{-\delta t_{n}} v\left(\widehat{c}_{s, t_{n}}^{*}\right) \Delta t .
$$

3. We solve for the optimal certainty equivalent consumption $c e^{*}$ :

$$
\frac{1}{\mathcal{S}} \sum_{s=1}^{\mathcal{S}} \sum_{n=0}^{\left\lfloor\frac{T}{\Delta t}\right\rfloor} e^{-\delta t_{n}} v\left(\widehat{c}_{s, t_{n}}^{*}\right) \Delta t=\sum_{n=0}^{\left\lfloor\frac{T}{\Delta t}\right\rfloor} e^{-\delta t_{n}} v\left(c e^{*}-\theta_{t_{n}}\left(c e^{*}\right)\right) \Delta t
$$

where

$$
\theta_{t_{n}}\left(c e^{*}\right)=\theta_{0} e^{-\alpha t_{n}}+\beta \sum_{i=0}^{n-1} e^{-\alpha\left(t_{n}-t_{i}\right)} c e^{*} \Delta t .
$$

4. We compute the CRRA consumption strategy $c_{\gamma, s, t_{n}}$ for $\gamma=0,0.1, \ldots, 99.9,100, s=$ 
$1, \ldots, \mathcal{S}$ and $n=0, \ldots,\left\lfloor\frac{T}{\Delta t}\right\rfloor$. The associated reference level can be computed as follows:

$$
\theta_{\gamma, s, t_{n}}=\theta_{0} e^{-\alpha t_{n}}+\beta \sum_{i=0}^{n-1} e^{-\alpha\left(t_{n}-t_{i}\right)} c_{\gamma, s, t_{n}} \Delta t
$$

so that the suboptimal dual consumption strategy is given by $\widehat{c}_{\gamma, s, t_{n}}=c_{\gamma, s, t_{n}}-\theta_{\gamma, s, t_{n}}$. Expected lifetime utility can now be approximated by

$$
\mathbb{E}\left[\int_{0}^{T} e^{-\delta t} v\left(\widehat{c}_{t}\right) \mathrm{d} t\right] \approx \frac{1}{\mathcal{S}} \sum_{s=1}^{\mathcal{S}} \sum_{n=0}^{\left\lfloor\frac{T}{\Delta t}\right\rfloor} e^{-\delta t_{n}} v\left(\widehat{c}_{\gamma, s, t_{n}}\right) \Delta t .
$$

5. We solve for the suboptimal certainty equivalent consumption $c e^{\gamma}$ for $\gamma=0,0.1, \ldots, 99.9,100$ :

$$
\frac{1}{\mathcal{S}} \sum_{s=1}^{\mathcal{S}} \sum_{n=0}^{\left\lfloor\frac{T}{\Delta t}\right\rfloor} e^{-\delta t_{n}} v\left(\widehat{c}_{\gamma, s, t_{n}}\right) \Delta t=\sum_{n=0}^{\left\lfloor\frac{T}{\Delta t}\right\rfloor} e^{-\delta t_{n}} v\left(c e^{\gamma}-\theta_{t_{n}}\left(c e^{\gamma}\right)\right) \Delta t
$$

where

$$
\theta_{t_{n}}\left(c e^{\gamma}\right)=\theta_{0} e^{-\alpha t_{n}}+\beta \sum_{i=0}^{n-1} e^{-\alpha\left(t_{n}-t_{i}\right)} c e^{\gamma} \Delta t .
$$

6. Finally, we compute the minimum welfare loss $m$ :

$$
m=\min _{\gamma \in\{0,0.1, \ldots, 99.9,100\}} \frac{c e^{*}-c e^{\gamma}}{c e^{*}} .
$$




\section{References}

Abdellaoui, M., Bleichrodt, H., Paraschiv, C., 2007. Loss aversion under prospect theory: A parameter-free measurement. Management Science 53, 1659-1674.

Abdellaoui, M., Vossmann, F., Weber, M., 2005. Choice-based elicitation and decomposition of decision weights for gains and losses under uncertainty. Management Science 51, 1384-1399.

Abel, A. B., 1990. Asset prices under habit formation and catching up with the Joneses. American Economic Review 80, 38-42.

Andries, M., 2012. Consumption-based asset pricing with loss aversion, Working Paper.

Ang, A., Bekaert, G., Lui, J., 2005. Why stocks may disappoint. Journal of Financial Economics $76,471-508$.

Barberis, N., Huang, M., 2008. Stocks as lotteries: The implications of probability weighting for security prices. American Economic Review 98, 2066-2100.

Barberis, N., Huang, M., Santos, T., 2001. Prospect theory and asset prices. Quarterly Journal of Economics 116, 1-53.

Barberis, N., Xiong, W., 2009. What drives the disposition effect? An analysis of a long-standing preference-based explanation. Journal of Finance 64, 751-784.

Basak, S., Shapiro, A., 2001. Value-at-risk based risk management: Optimal policies and asset prices. Review of Financial Studies 14, 371-405.

Bell, D. E., 1982. Regret in decision making under uncertainty. Operations Research 30, 961981.

Bell, D. E., 1983. Risk premiums for decision regret. Management Science 29, 1156-1166.

Bell, D. E., 1985. Disappointment in decision making under uncertainty. Operations Research $33,1-27$.

Benartzi, S., Thaler, R. H., 1995. Myopic loss aversion and the equity premium puzzle. Quarterly Journal of Economics 110, 73-92.

Benzoni, L., Collin-Dufresne, P., Goldstein, R. S., 2007. Portfolio choice over the life-cycle when the stock and labor markets are cointegrated. Journal of Finance 62, 2123-2167.

Berkelaar, A. B., Kouwenberg, R., Post, T., 2004. Optimal portfolio choice under loss aversion. Review of Economics and Statistics 86, 973-987.

van Bilsen, S., 2015. Essays on Intertemporal Consumption and Portfolio Choice. Tilburg: CentER, Center for Economic Research.

Bodie, Z., Detemple, J. B., Otruba, S., Walter, S., 2004. Optimal consumption-portfolio choices and retirement planning. Journal of Economic Dynamics and Control 28, 1115-1148.

Bodie, Z., Merton, R. C., Samuelson, W. F., 1992. Labor supply flexibility and portfolio choice in a life-cycle model. Journal of Economic Dynamics and Control 16, 427-449.

Booij, A. S., van de Kuilen, G., 2009. A parameter-free analysis of the utility of money for the general population under prospect theory. Journal of Economic Psychology 30, 651-666.

Booij, A. S., van Praag, B. M. S., van de Kuilen, G., 2010. A parametric analysis of prospect theory's functionals for the general population. Theory and Decision 68, 115-148.

Campbell, J. Y., Cocco, J., Gomes, F., Maenhout, P. J., Viceira, L. M., 2001. Stock market mean reversion and the optimal equity allocation of a long-lived investor. European Finance Review 5, 269-292. 
Campbell, J. Y., Deaton, A., 1989. Why is consumption so smooth? Review of Economic Studies 56, 357-373.

Carlson, M., Lazrak, A., 2014. Household wealth and portfolio choice when tail events are salient, Working Paper.

Chacko, G., Viceira, L. M., 2005. Dynamic consumption and portfolio choice with stochastic volatility in incomplete markets. Review of Financial Studies 18, 1369-1402.

Chew, S. H., Karni, E., Safra, Z., 1987. Risk aversion in the theory of expected utility with rank dependent probabilities. Journal of Economic Theory 42, 370-381.

Cocco, J. F., Gomes, F. J., Maenhout, P. J., 2005. Consumption and portfolio choice over the life cycle. Review of Financial Studies 18, 491-533.

Constantinides, G. M., 1990. Habit formation: A resolution of the equity premium puzzle. Journal of Political Economy 98, 519-543.

Cox, J. C., Huang, C., 1989. Optimal consumption and portfolio policies when asset prices follow a diffusion process. Journal of Economic Theory 49, 33-83.

Cox, J. C., Huang, C., 1991. A variational problem arising in financial economics. Journal of Mathematical Economics 20, 465-487.

Curatola, G., 2015. Loss aversion, habit formation and the term structures of equity and interest rates. Journal of Economic Dynamics and Control 53, 103-122.

Curatola, G., 2017. Optimal portfolio choice with loss aversion over consumption. The Quarterly Review of Econimics and Finance 66, 345-358.

Deaton, A., 1987. Life-cycle models of consumption: Is the evidence consistent with the theory? In: Bewley, T. F. (ed.), Advances in Econometrics: Fifth World Congress, Cambridge University Press, vol. 2, pp. 121-148.

Deelstra, G., Grasselli, M., Koehl, P.-F., 2003. Optimal investment strategies in the presence of a minimum guarantee. Insurance: Mathematics and Economics 33, 189-207.

Dynan, K. E., Skinner, J., Zeldes, S. P., 2004. Do the rich save more? Journal of Political Economy 2, 397-444.

Etchart-Vincent, N., 2004. Is probability weighting sensitive to the magnitude of consequences? An empirical investigation on losses. Journal of Risk and Uncertainty 28, 217-235.

Fagereng, A., Gottlieb, C., Guiso, L., 2017. Asset market participation and portfolio choice over the life-cycle. Journal of Finance 72, 705-750.

Flavin, M., 1985. Excess sensitivity of consumption to current income: Liquidity constraints or myopia? Canadian Journal of Economics 18, 117-136.

Gomes, F., Michaelides, A., 2003. Portfolio choice with internal habit formation: A life-cycle model with uninsurable labor income risk. Review of Economic Dynamics 6, 729-766.

Gomes, F., Michaelides, A., 2005. Optimal life-cycle asset allocation: Understanding the empirical evidence. Journal of Finance 60, 869-904.

Gomes, F. J., 2005. Portfolio choice and trading volume with loss averse investors. Journal of Business 78, 675-706.

Gomes, F. J., Kotlikoff, L. J., Viceira, L. M., 2008. Optimal life-cycle investing with flexible labor supply: A welfare analysis of life-cycle funds. American Economic Review 98, 297-303.

Guasoni, P., Huberman, G., Ren, D., 2015. Shortfall aversion, Working Paper. 
Guillén, M., Jørgensen, P. L., Nielsen, J. P., 2006. Return smoothing mechanisms in life and pension insurance: Path-dependent contingent claims. Insurance: Mathematics and Economics 38, 229-252.

Guillén, M., Nielsen, J. P., Pérez-Marín, A. M., Petersen, K. S., 2013. Performance measurement of pension strategies: a case study of danish life-cycle products. Scandinavian Actuarial Journal 2013, 49-68.

Gul, F., 1991. A theory of disappointment aversion. Econometrica 59, 667-686.

He, X. D., Zhou, X. Y., 2011. Portfolio choice via quantiles. Mathematical Finance 21, 203-231.

He, X. D., Zhou, X. Y., 2016. Hope, fear and aspirations. Mathematical Finance 26, 3-50.

Jin, H., Zhou, X. Y., 2008. Behavioral portfolio selection in continuous time. Mathematical Finance 18, 385-426.

Johnson, D. S., Parker, J. A., Souleles, N. S., 2006. Household expenditure and the income tax rebates of 2001. American Economic Review 5, 1589-1610.

Jørgensen, P. L., Linnemann, P., 2011. A comparison of three different pension savings products with special emphasis on the payout phase. Annals of Actuarial Science 6, 137-152.

Kahneman, D., Tversky, A., 1979. Prospect theory: An analysis of decision under risk. Econometrica 47, 263-292.

Karatzas, I., Lehoczky, J. P., Shreve, S. E., 1987. Optimal consumption and portfolio decisions for a "small investor" on a finite horizon. SIAM Journal of Control and Optimization 25, $1557-1586$.

Karatzas, I., Shreve, S. E., 1998. Methods of Mathematical Finance, vol. 39. Springer.

Kőszegi, B., Rabin, M., 2006. A model of reference-dependent preferences. Quarterly Journal of Economics 121, 1133-1165.

Köszegi, B., Rabin, M., 2007. Reference-dependent risk attitudes. American Economic Review 97, 1047-1073.

Kőszegi, B., Rabin, M., 2009. Reference-dependent consumption plans. American Economic Review 99, 909-936.

Laeven, R. J. A., Stadje, M. A., 2014. Robust portfolio choice and indifference valuation. Mathematics of Operations Research 39, 1109-1141.

Linnemann, P., Bruhn, K., Steffensen, M., 2014. A comparison of modern investment-linked pension savings products. Annals of Actuarial Science 9, 72-84.

Liu, J., 2007. Portfolio selection in stochastic environments. Review of Financial Studies 20, $1-39$.

Loomes, G., Sugden, R., 1982. Regret theory: An alternative theory of rational choice under uncertainty. Economic Journal 92, 805-824.

Loomes, G., Sugden, R., 1986. Disappointment and dynamic consistency in choice under uncertainty. Review of Economic Studies 53, 271-282.

Mankiw, N. G., Zeldes, S. P., 1991. The consumption of stockholders and nonstockholders. Journal of Financial Economics 29, 97-112.

Maurer, R., Mitchell, O. S., Rogalla, R., Siegelin, I., 2016. Accounting and actuarial smoothing of retirement payouts in participating life annuities. Insurance: Mathematics and Economics $71,268-283$. 
Meng, J., Weng, X., 2017. Can prospect theory explain the disposition effect? A new perspective on reference points. Management Science (Forthcoming).

Merton, R. C., 1969. Lifetime portfolio selection under uncertainty: The continuous-time case. Review of Economics and Statistics 51, 247-257.

Morningstar, 2017. 2017 target-date fund landscape, answers to frequently asked questions.

Mossin, J., 1968. Optimal multiperiod portfolio policies. Journal of Business 41, 215-229.

Muermann, A., Mitchell, O. S., Volkman, J. M., 2006. Regret, portfolio choice, and guarantees in defined contribution schemes. Insurance: Mathematics and Economics 39, 219-229.

Munk, C., 2008. Portfolio and consumption choice with stochastic investment opportunities and habit formation in preferences. Journal of Economic Dynamics and Control 32, 3560-3589.

Odean, T., 1998. Are investors reluctant to realize their losses? Journal of Finance 53, 17751798.

Pagel, M., 2017a. Expectations-based reference-dependent life-cycle consumption. Review of Economic Studies 84, 885-934.

Pagel, M., 2017b. Expectations-based reference-dependent preferences and asset pricing. Journal of the European Economic Association (Forthcoming).

Pliska, S. R., 1986. A stochastic calculus model of continuous trading: Optimal portfolios. Mathematics of Operations Research 11, 371-382.

Quiggin, J., 1994. Regret theory with general choice sets. Journal of Risk and Uncertainty 8, $153-165$.

Samuelson, P. A., 1969. Lifetime portfolio selection by dynamic stochastic programming. Review of Economics and Statistics 51, 239-246.

Samwick, A. A., 1998. Discount rate heterogeneity and social security reform. Journal of Development Economics 57, 117-146.

Schroder, M., Skiadas, C., 2002. An isomorphism between asset pricing models with and without linear habit formation. Review of Financial Studies 15, 1189-1221.

Shefrin, H., Statman, M., 1985. The disposition to sell winners too early and ride losers too long: Theory and evidence. Journal of Finance 40, 777-790.

Shumway, T., 1997. Explaining returns with loss aversion, Working Paper.

Sugden, R., 1993. An axiomatic foundation for regret theory. Journal of Economic Theory 60, $159-180$.

Tversky, A., Kahneman, D., 1992. Advances in prospect theory: Cumulative representation of uncertainty. Journal of Risk and Uncertainty 5, 297-323.

Viceira, L. M., 2001. Optimal portfolio choice for long-horizon investors with nontradable labor income. Journal of Finance 56, 433-470.

Vissing-Jørgensen, A., 2002. Limited asset market participation and the elasticity of intertemporal substitution. Journal of Political Economy 110, 825-853.

Wachter, J. A., 2002. Portfolio and consumption decisions under mean-reverting returns: An exact solution for complete markets. Journal of Financial and Quantitative Analysis 37, 6391.

Yogo, M., 2008. Asset prices under habit formation and reference-dependent preferences. Journal of Business and Economic Statistics 26, 539-558. 


\section{Figures \& Table}

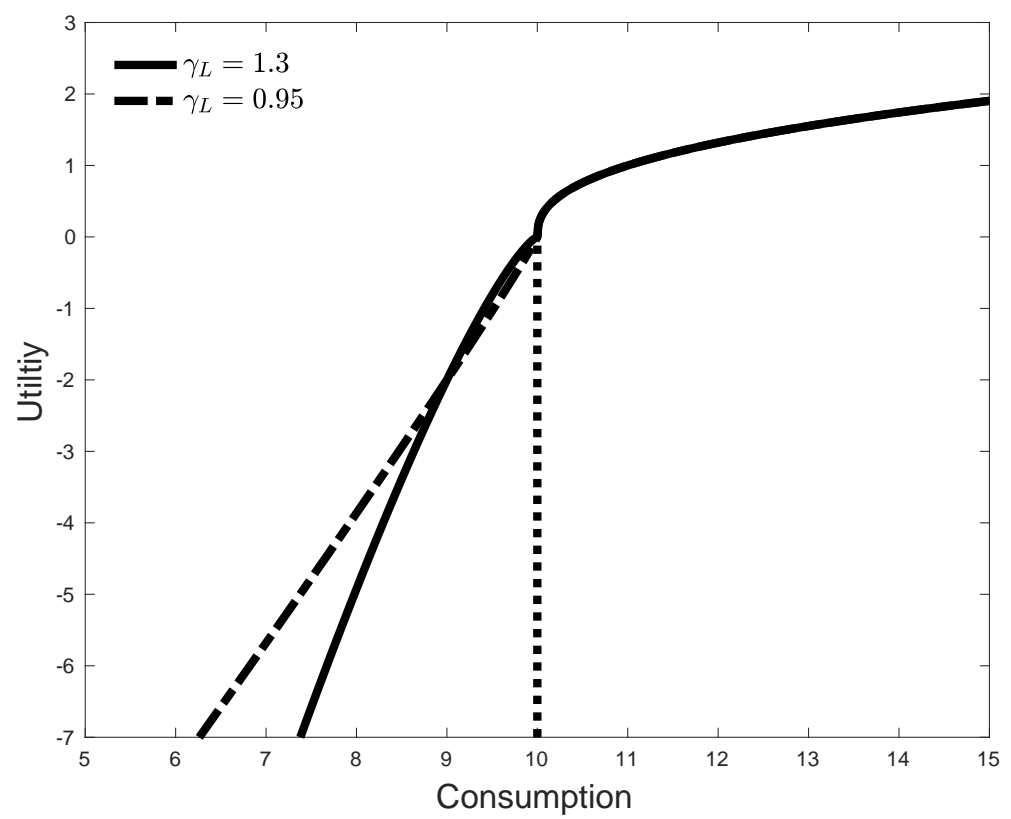

Figure 1: Illustration of the two-part power utility function. The figure illustrates the two-part power utility function for two different values of the curvature parameter for losses $\gamma_{L}$. The individual's reference level is set equal to 10 , the loss aversion parameter $\kappa$ to 2 , and the curvature parameter for gains $\gamma_{G}$ to 0.4 . 
Consumption choice:

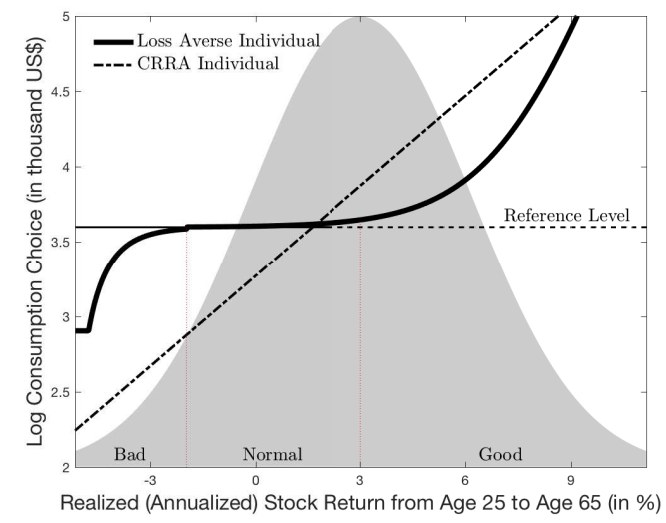

(a) Risk averse for losses

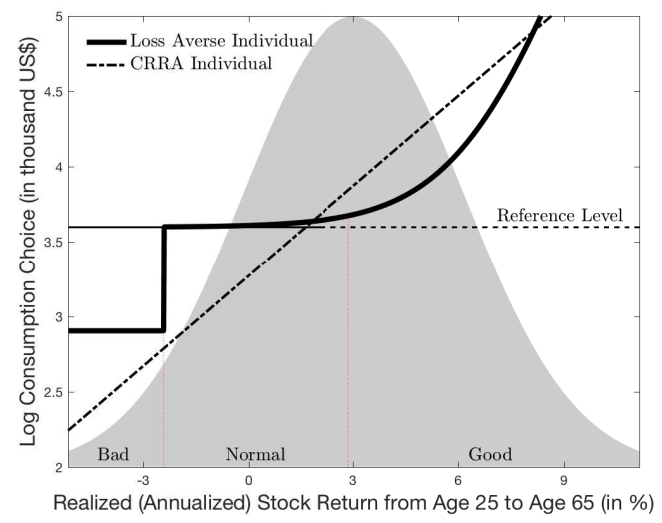

(b) Risk loving for losses

Consumption-to-total-wealth ratio:

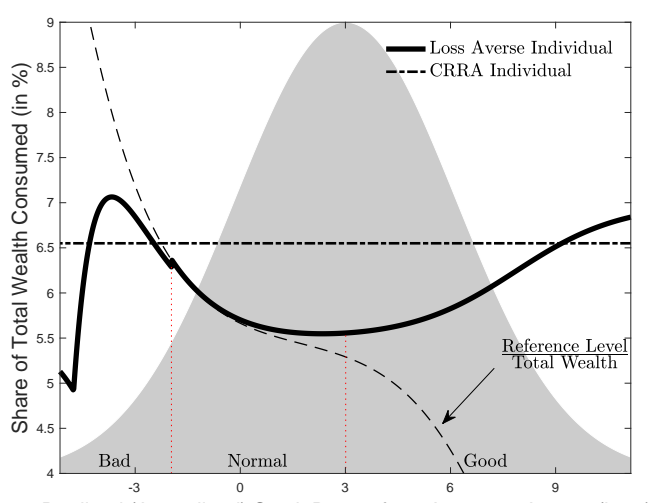

Realized (Annualized) Stock Return from Age 25 to Age 65 (in \%)

(c) Risk averse for losses

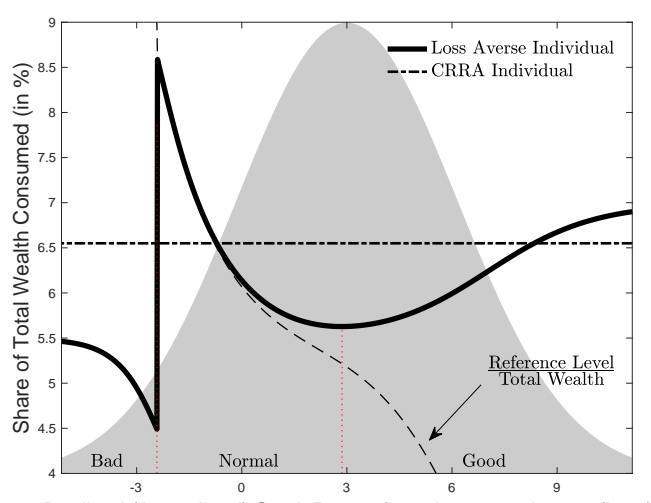

Realized (Annualized) Stock Return from Age 25 to Age 65 (in \%)

(d) Risk loving for losses

Share of total wealth invested in the stock:

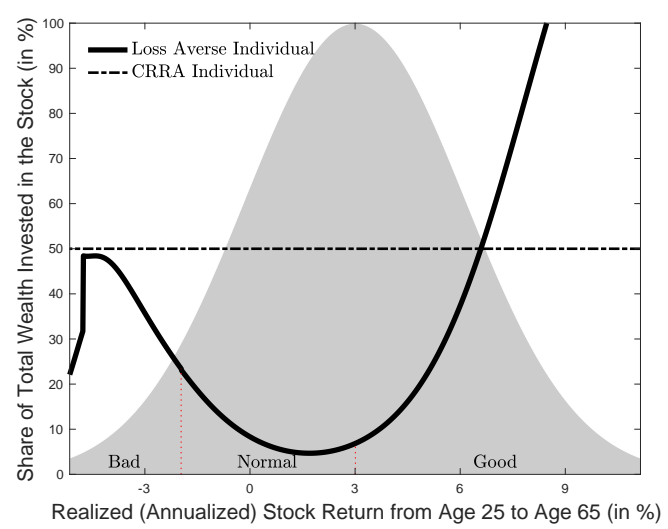

(e) Risk averse for losses

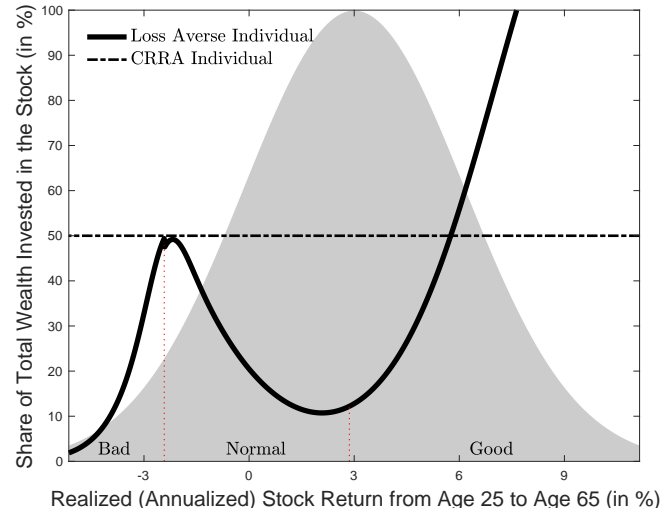

(f) Risk loving for losses

Figure 2: Optimal behavior of a loss-averse individual. The figure shows the optimal current behavior of a 65-year-old loss-averse individual as a function of the annualized stock return from $t=0$ (age 25) to $t=40$ (age 65). The left panels assume risk-averse behavior in the loss domain $\left(\gamma_{L}=1.3\right)$, whereas the right panels assume risk-loving behavior in the loss domain $\left(\gamma_{L}=0.95\right)$. The dash-dotted lines show the behavior of a CRRA individual with relative risk aversion equal to 2 . The parameter $L_{t}^{\max }-$ which denotes the maximum amount by which consumption can fall below the reference level - is set equal to $50 \%$ of the reference level. The gray areas represent the probability density function of the annualized stock return conditional upon information available at age 25 . 
Medium-wealth individuals:

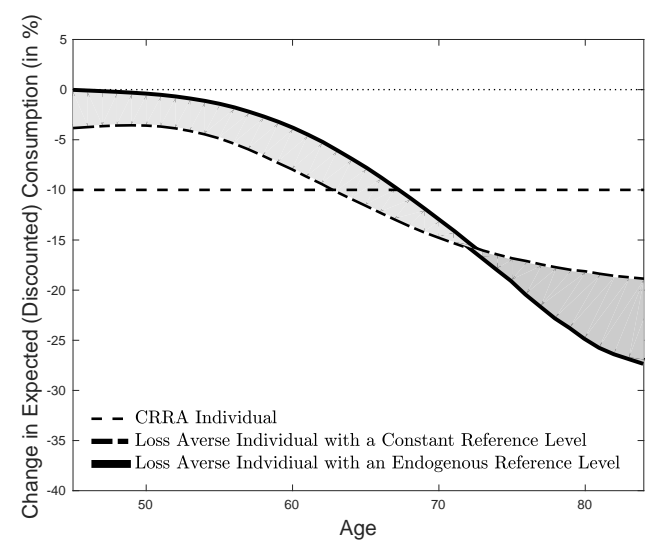

(a) Risk averse for losses

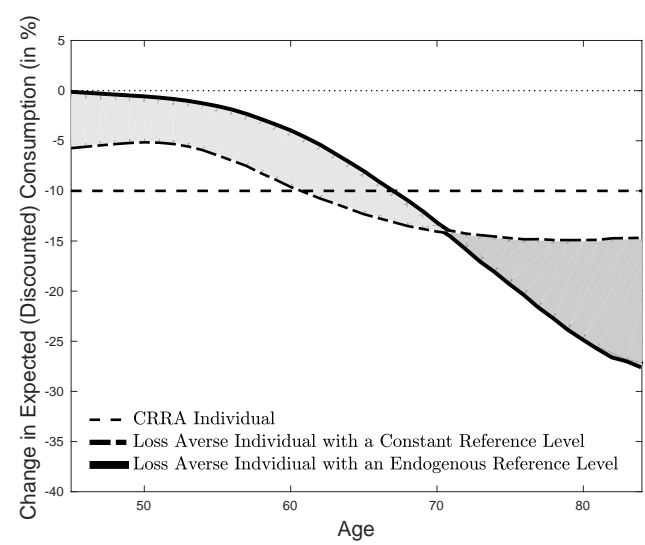

(b) Risk loving for losses

High-wealth individuals:

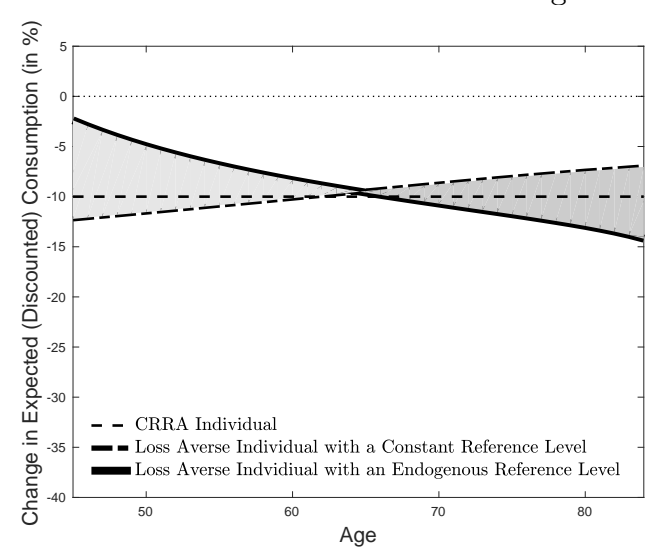

(c) Risk averse for losses

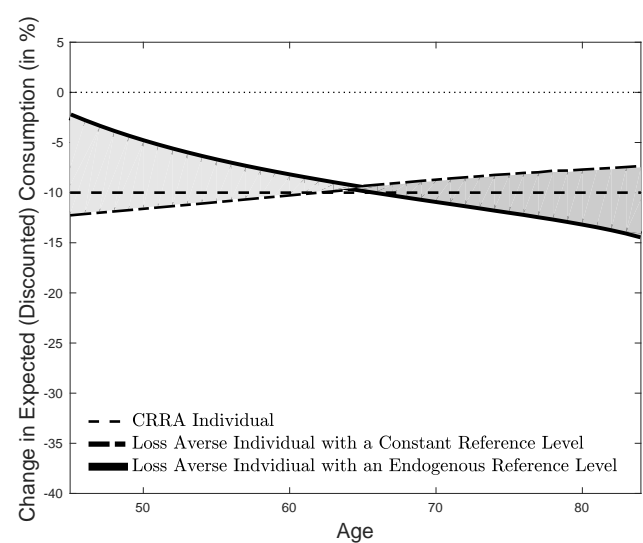

(d) Risk loving for losses

Low-wealth individuals:

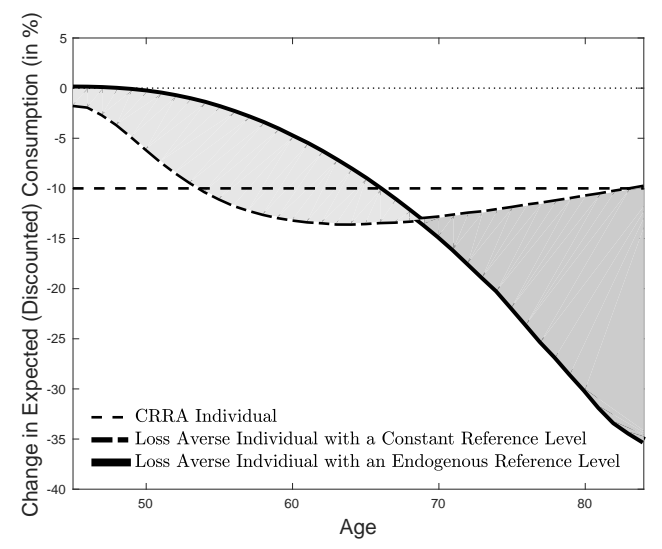

(e) Risk averse for losses

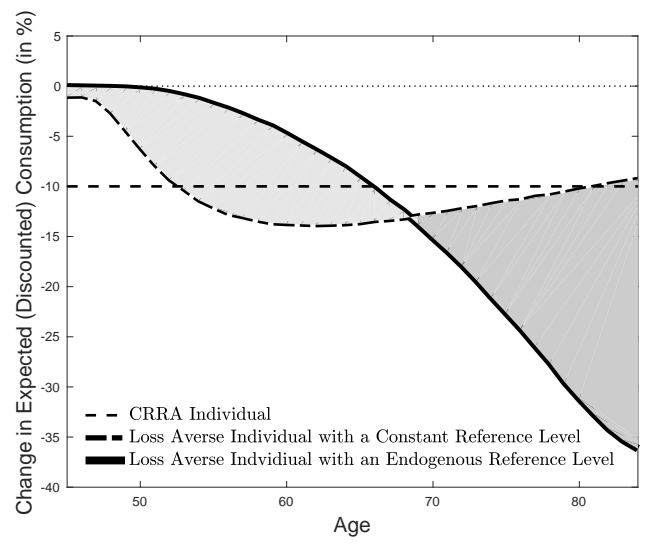

(f) Risk loving for losses

Figure 3: Shock absorbing mechanisms. The figure shows the impact of a $10 \%$ drop in the individual's total wealth at age 45 on expected (discounted) consumption for medium-wealth individuals (upper panels), for high-wealth individuals (middle panels), and for low-wealth individuals (lower panels). The value of the reference level at age 45 is the same in all six panels. The upper, middle and lower panels assume, respectively, that, before the wealth shock is realized, the individual's total wealth at age 45 is $100 \%, 200 \%$ and $85 \%$ of the costs of financing a consumption stream that is equal to the reference level at age 45 . The left panels assume risk-averse behavior in the loss domain $\left(\gamma_{L}=1.3\right)$, whereas the right panels assume risk-loving behavior in the loss domain $\left(\gamma_{L}=0.95\right)$. The parameter $L_{t}^{\max }-$ which denotes the maximum amount by which consumption can fall below the reference level - is set equal to $50 \%$ of the initial reference level. 
Portfolio choice expressed as share of total wealth:

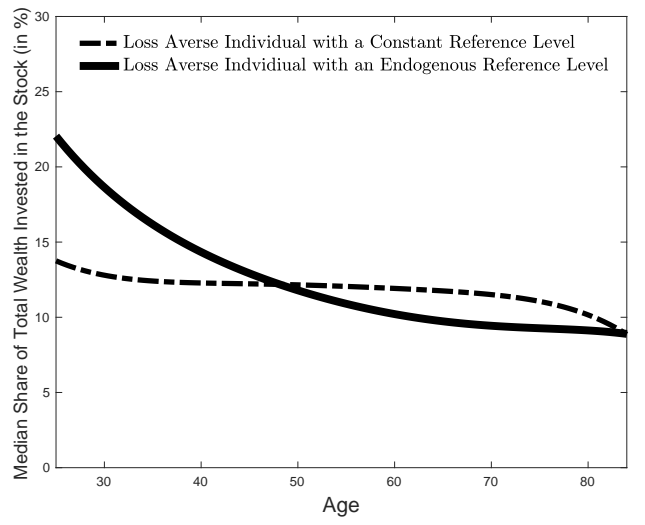

(a) Risk averse for losses

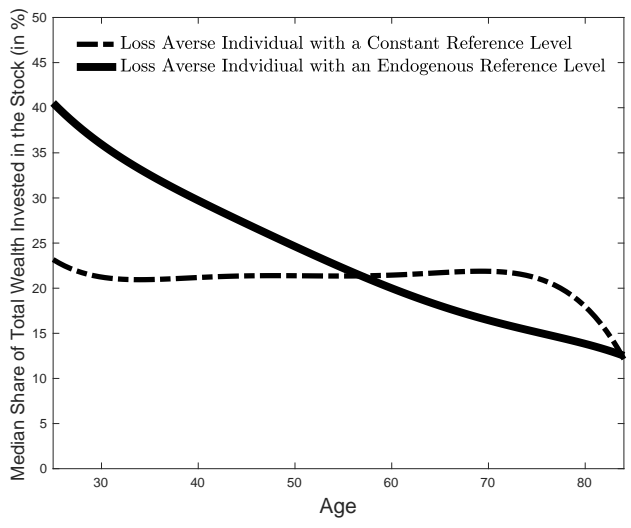

(b) Risk loving for losses

Portfolio choice expressed as share of financial wealth:

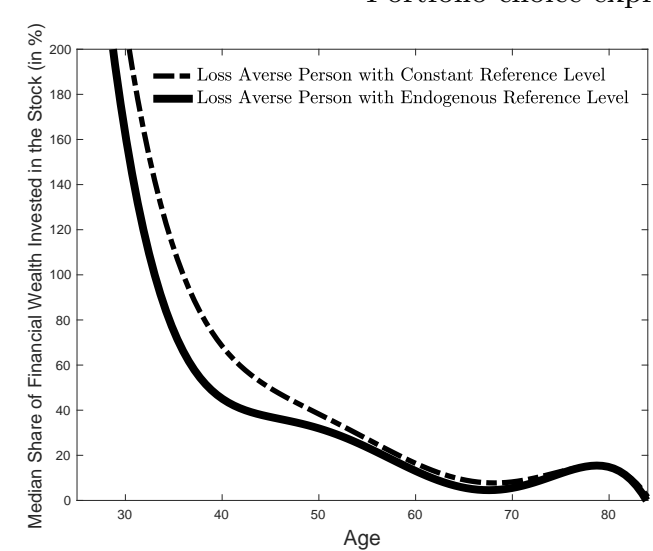

(c) Risk averse for losses

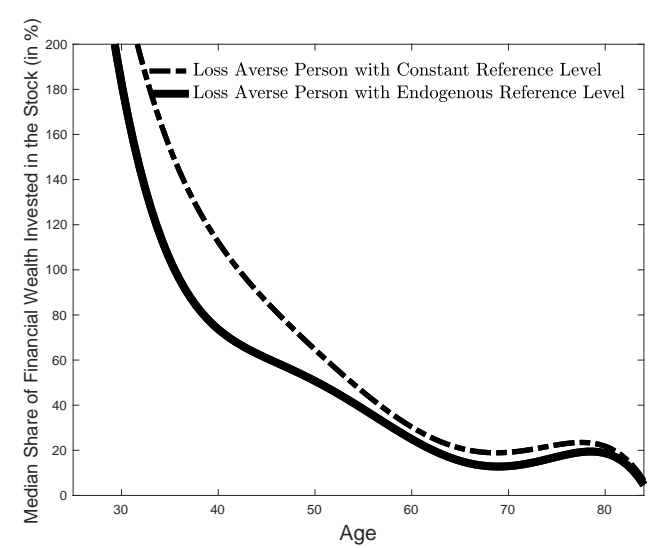

(d) Risk loving for losses

Median wealth accumulation:

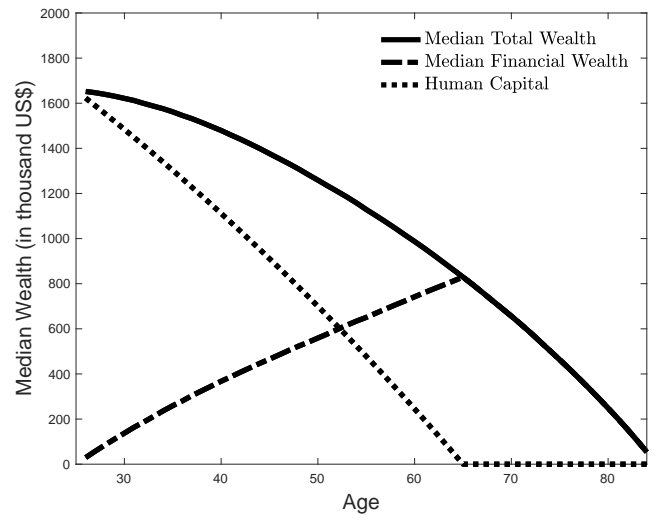

(e) Risk averse for losses

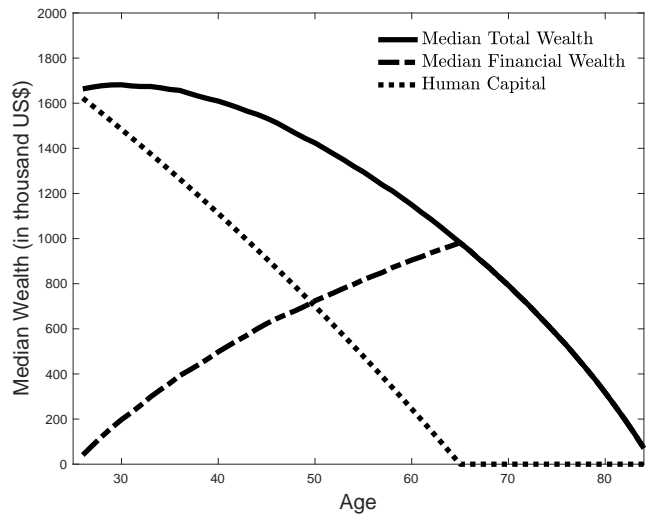

(f) Risk loving for losses

Figure 4: Median optimal portfolio choice. The figure shows the median share of wealth invested in the stock as a function of age. The upper panels illustrate the portfolio strategy in terms of total wealth, whereas the middle panels illustrate the portfolio strategy in terms of financial wealth. The lower panels show the median wealth accumulation for a loss-averse individual with an endogenous reference level. The parameter $L_{t}^{\max }-$ which denotes the maximum amount by which consumption can fall below the reference level - is set equal to $50 \%$ of the initial reference level. The left panels assume risk-averse behavior in the loss domain $\left(\gamma_{L}=1.3\right)$, whereas the right panels assume risk-loving behavior in the loss domain $\left(\gamma_{L}=0.95\right)$. 


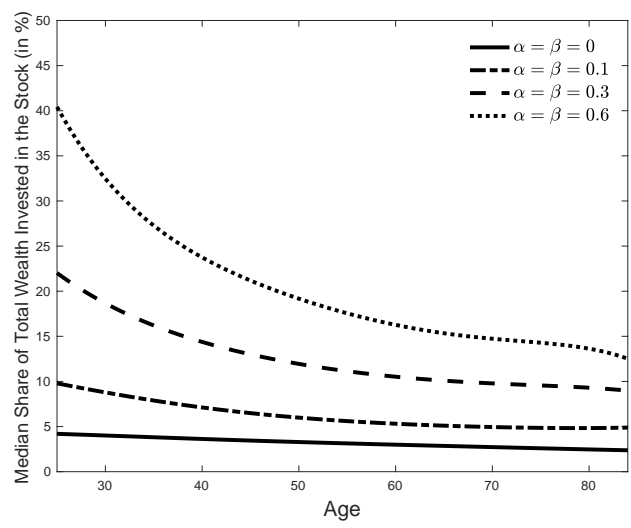

(a) Risk averse for losses

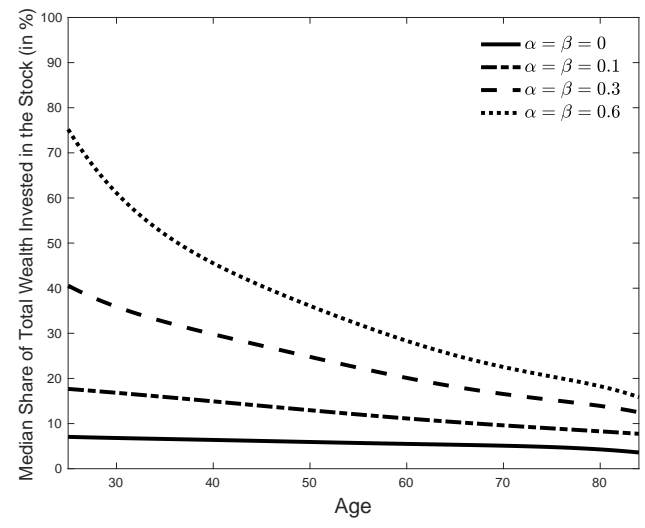

(b) Risk loving for losses

Figure 5: Median optimal portfolio choice. The figure shows the median share of total wealth invested in the stock for various values of $\alpha$ and $\beta$. The left figure assumes risk-averse behavior in the loss domain $\left(\gamma_{L}=1.3\right)$, whereas the right figure assumes risk-loving behavior in the loss domain $\left(\gamma_{L}=0.95\right)$. The other parameter values are as follows: $\left(\kappa, \gamma_{G}\right)=(5,0.2)$. The parameter $L_{t}^{\max }-$ which denotes the maximum amount by which consumption can fall below the reference level - is set equal to $50 \%$ of the initial reference level. 


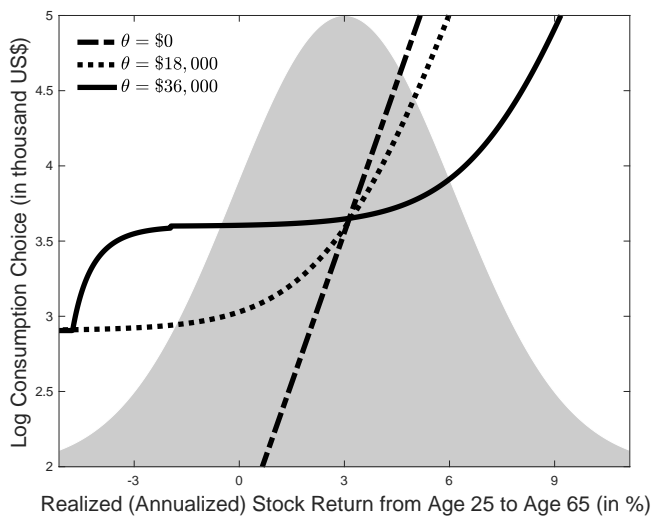

(a) Change in the reference level

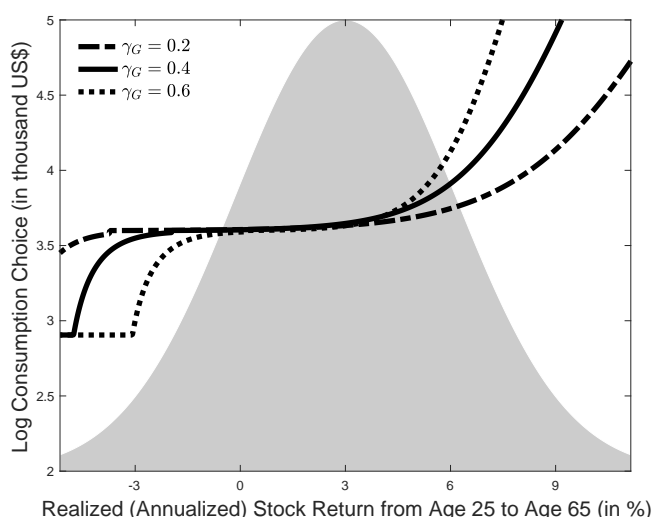

(c) Change in the curvature parameter for gains

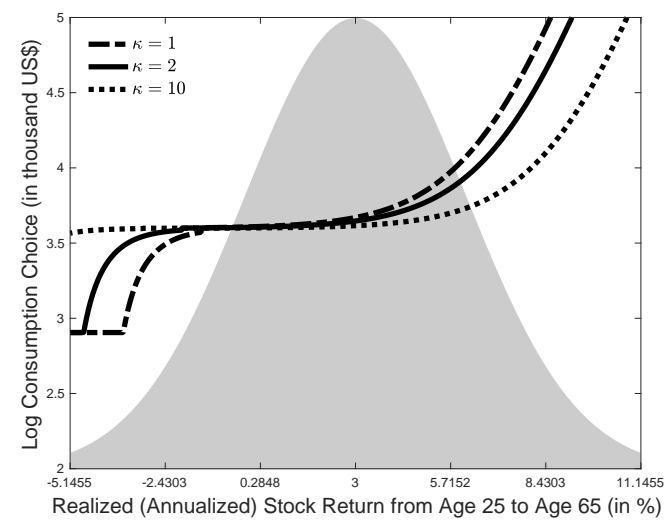

(b) Change in the loss aversion parameter

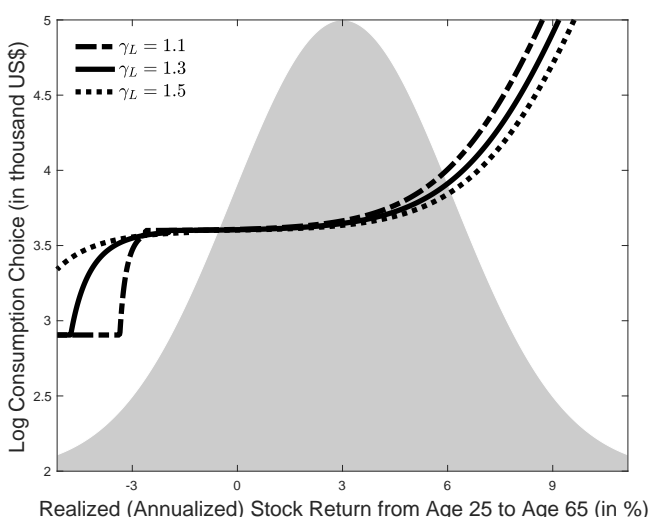

(d) Change in the curvature parameter for losses

Figure 6: Optimal consumption behavior of a loss-averse individual for various values of the preference parameters. The figure shows the optimal current consumption behavior of a 65-year-old lossaverse individual as a function of the annualized stock return from $t=0$ (i.e., age 25) to $t=40$ (i.e., age 65). The solid lines correspond to the benchmark case (see also Figure 2(a)). The benchmark parameter values are as follows: $\left(\theta, \kappa, \gamma_{G}, \gamma_{L}, \alpha, \beta\right)=(\$ 36000,2,0.4,1.3,0,0)$. The parameter $L_{t}^{\text {max }}$ - which denotes the maximum amount by which consumption can fall below the reference level - is set equal to $50 \%$ of the (initial) reference level. The gray areas represent the probability density function of the annualized stock return conditional upon information available at age 25 . 


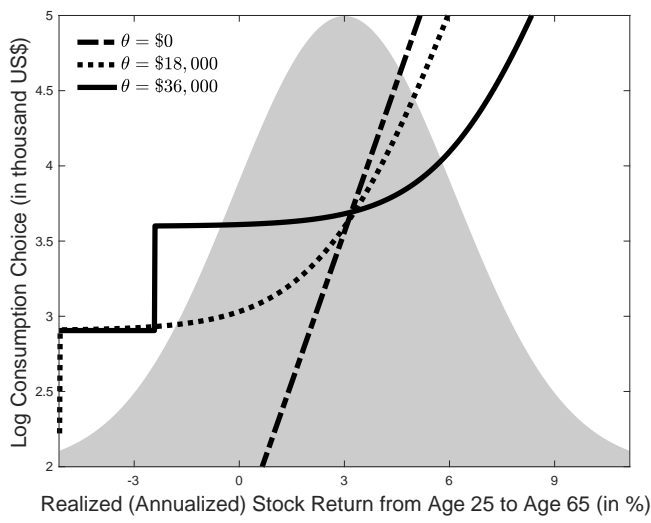

(a) Change in the reference level

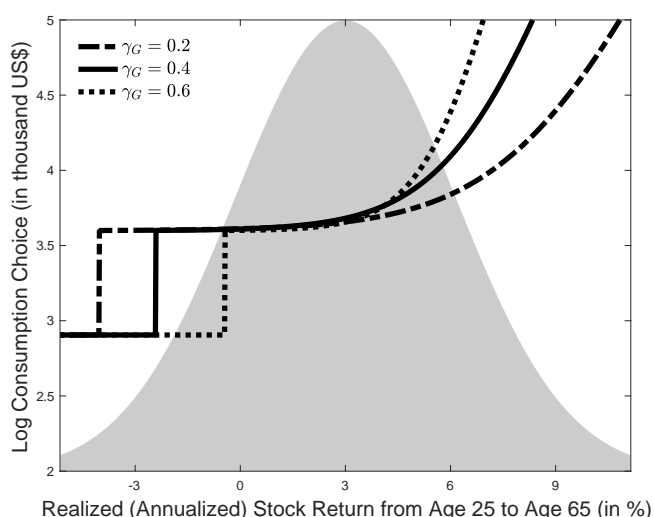

(c) Change in the curvature parameter for gains

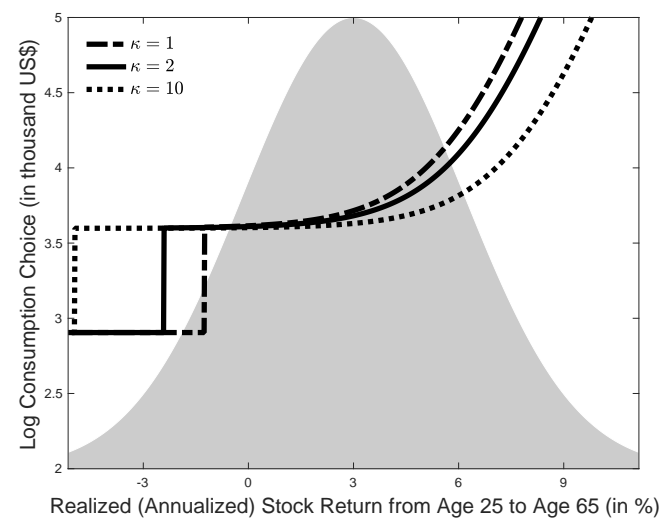

(b) Change in the loss aversion parameter

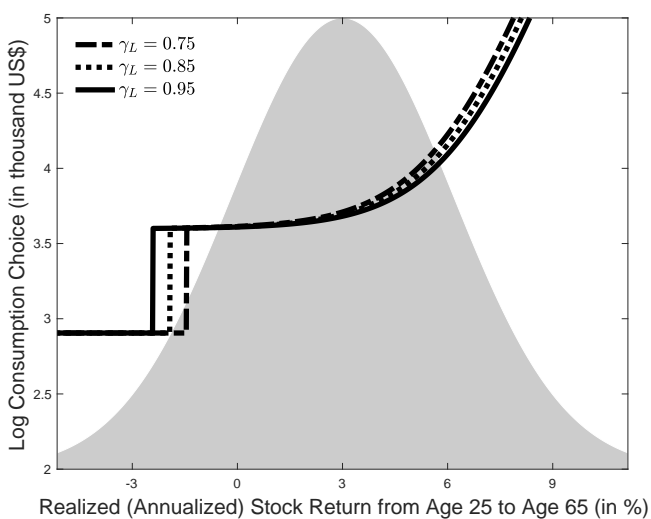

(d) Change in the curvature parameter for losses

Figure 7: Optimal consumption behavior of a loss-averse individual for various values of the preference parameters. The figure shows the optimal consumption behavior of a 65-year-old loss-averse individual as a function of the annualized stock return from $t=0$ (i.e., age 25) to $t=40$ (i.e., age 65). The solid lines correspond to the benchmark case (see also Figure 2(b)). The benchmark parameter values are as follows: $\left(\theta, \kappa, \gamma_{G}, \gamma_{L}, \alpha, \beta\right)=(\$ 36000,2,0.4,0.95,0,0)$. The parameter $L_{t}^{\max }$ - which denotes the maximum amount by which consumption can fall below the reference level - is set equal to $50 \%$ of the (initial) reference level. The gray areas represent the probability density function of the annualized stock return conditional upon information available at age 25 . 
Table 1:

\section{Consumption and Portfolio Dynamics}

The table reports, for various ages, the (unconditional) probability that i) consumption is lower than the reference level (second column), ii) consumption exceeds the reference level by more than $10 \%$ (third column), and iii) the share of total wealth invested in the stock is less than $50 \%$ (third column). The benchmark parameter values are as follows: $\left(\theta, \kappa, \gamma_{G}, \alpha, \beta\right)=(\$ 36000,2,0.4,0,0)$. The upper table considers risk-averse behavior in the loss domain $\left(\gamma_{L}=1.3\right)$, whereas the lower table considers risk-loving behavior in the loss domain $\left(\gamma_{L}=0.95\right)$. We set the parameter $L_{t}^{\max }$ - which denotes the maximum amount by which consumption can fall below the reference level - to $50 \%$ of the initial reference level.

(a) Risk Averse for Losses

\begin{tabular}{cccc}
\hline Age & $\mathbb{P}\left[c_{t}^{*}<\theta_{t}^{*}\right]($ in $\%)$ & $\mathbb{P}\left[c_{t}^{*}>1.1 \times \theta_{t}^{*}\right]($ in $\%)$ & $\mathbb{P}\left[\pi_{t}^{*} / W_{t}^{*}<0.5\right]($ in $\%)$ \\
\hline 35 & 0.0 & 46.4 & 97.9 \\
45 & 2.7 & 45.3 & 94.0 \\
55 & 13.1 & 44.4 & 90.6 \\
65 & 19.6 & 43.6 & 87.9 \\
75 & 24.2 & 42.9 & 83.0 \\
\hline
\end{tabular}

(b) Risk Loving for Losses

\begin{tabular}{cccc}
\hline Age & $\mathbb{P}\left[c_{t}^{*}<\theta_{t}^{*}\right]($ in $\%)$ & $\mathbb{P}\left[c_{t}^{*}>1.1 \times \theta_{t}^{*}\right]($ in $\%)$ & $\mathbb{P}\left[\pi_{t}^{*} / W_{t}^{*}<0.5\right]$ (in \%) \\
\hline 35 & 0.0 & 56.6 & 93.3 \\
45 & 0.0 & 52.5 & 86.4 \\
55 & 9.9 & 50.3 & 83.5 \\
65 & 16.8 & 48.7 & 81.0 \\
75 & 21.7 & 47.5 & 73.6 \\
\hline
\end{tabular}




\section{Table 2:}

\section{Shock Absorbing Mechanisms}

The table shows, for various degrees of endogeneity, the impact of a $10 \%$ drop in the individual's total wealth at age 45 on expected (discounted) consumption for medium-wealth individuals (panels (a) and (b)) and for lowwealth individuals (panels (c) and (d)). The value of the reference level at age 45 is the same in all four panels. Panels (a) and (b) (respectively, (c) and (d)) assume that, before the wealth shock is realized, the individual's total wealth at age 45 is $100 \%$ (respectively, 85\%) of the costs of financing a consumption stream that is equal to the reference level at age 45. Panels (a) and (c) assume risk-averse behavior in the loss domain $\left(\gamma_{L}=1.3\right)$, whereas panels (b) and (d) assume risk-loving behavior in the loss domain $\left(\gamma_{L}=0.95\right)$. The parameter $L_{t}^{\max }-$ which denotes the maximum amount by which consumption can fall below the reference level - is set equal to $50 \%$ of the initial reference level. The other parameter values are as follows: $\left(\kappa, \gamma_{G}\right)=(5,0.2)$.

(a) Medium Wealth and Risk Averse for Losses

\begin{tabular}{ccccc}
\hline & \multicolumn{3}{c}{$\beta=\alpha$} \\
\cline { 2 - 5 } Horizon & 0 & 0.1 & 0.3 & 0.6 \\
\hline 1 & -1.29 & -0.39 & -0.22 & -0.15 \\
5 & -1.23 & -0.60 & -0.50 & -0.50 \\
10 & -2.28 & -1.73 & -1.32 & -1.22 \\
20 & -9.81 & -8.40 & -7.26 & -6.52 \\
40 & -24.05 & -26.72 & -28.38 & -29.07 \\
\hline
\end{tabular}

(b) Medium Wealth and Risk Loving for Losses

\begin{tabular}{|c|c|c|c|c|}
\hline \multirow[b]{2}{*}{ Horizon } & \multicolumn{4}{|c|}{$\beta=\alpha$} \\
\hline & 0 & 0.1 & 0.3 & 0.6 \\
\hline 1 & -2.25 & -0.63 & -0.32 & -0.19 \\
\hline 5 & -1.99 & -0.85 & -0.68 & -0.63 \\
\hline 10 & -2.54 & -1.83 & -1.42 & -1.36 \\
\hline 20 & -10.34 & -8.62 & -7.37 & -6.79 \\
\hline 40 & -22.24 & -26.53 & -27.54 & -29.40 \\
\hline
\end{tabular}

(c) Low Wealth and Risk Averse for Losses

\begin{tabular}{ccccc}
\hline & \multicolumn{3}{c}{$\beta=\alpha$} \\
\cline { 2 - 5 } Horizon & 0 & 0.1 & 0.3 & 0.6 \\
\hline 1 & -1.30 & -0.03 & -0.04 & -0.04 \\
5 & -4.54 & -0.96 & -0.28 & -0.18 \\
10 & -10.21 & -3.44 & -1.58 & -1.00 \\
20 & -13.50 & -10.11 & -8.31 & -7.17 \\
40 & -10.02 & -28.76 & -35.40 & -40.63 \\
\hline
\end{tabular}

(d) Low Wealth and Risk Loving for Losses

\begin{tabular}{|c|c|c|c|c|}
\hline \multirow[b]{2}{*}{ Horizon } & \multicolumn{4}{|c|}{$\beta=\alpha$} \\
\hline & 0 & 0.1 & 0.3 & 0.6 \\
\hline 1 & -0.15 & -0.10 & -0.09 & -0.08 \\
\hline 5 & -3.35 & -0.43 & -0.22 & -0.28 \\
\hline 10 & -10.87 & -3.24 & -1.40 & -1.00 \\
\hline 20 & -14.14 & -10.21 & -8.01 & -7.08 \\
\hline 40 & -9.86 & -29.31 & -36.62 & -42.69 \\
\hline
\end{tabular}


Table 3:

\section{Minimum Welfare Losses}

The table reports the effect of a change in the individual's preference parameters on the minimum welfare loss. We measure welfare losses in terms of the relative decline in certainty equivalent consumption. The benchmark parameter values are as follows: $\left(\theta, \kappa, \gamma_{G}, \gamma_{L}, \alpha, \beta\right)=(\$ 36000,2,0.4,1.3,0,0)$. We set the parameter $L_{t}^{\max }-$ which denotes the maximum amount by which consumption can fall below the reference level - to $50 \%$ of the initial reference level. The last column denotes the value of the CRRA parameter that minimizes the difference between the individual's actual utility and the individual's optimal utility.

(a) Change in the Reference Level

\begin{tabular}{ccc}
\hline$\theta($ in US\$) & Welfare Loss (in \%) & CRRA Parameter \\
\hline 0 & 0 & 0.6 \\
9,000 & 11.48 & 1.3 \\
18,000 & 13.84 & 2.4 \\
27,000 & 9.60 & 4.9 \\
$\mathbf{3 6 , 0 0 0}$ & $\mathbf{2 . 9 6}$ & $\mathbf{1 4 . 5}$ \\
\hline
\end{tabular}

(b) Change in the Loss Aversion Parameter

\begin{tabular}{ccc}
\hline$\kappa$ & Welfare Loss (in \%) & CRRA Parameter \\
\hline 1 & 3.35 & 7.3 \\
1.5 & 3.19 & 10.8 \\
$\mathbf{2}$ & $\mathbf{2 . 9 6}$ & $\mathbf{1 4 . 5}$ \\
5 & 2.04 & 37.8 \\
10 & 1.40 & 80.1 \\
\hline
\end{tabular}

(c) Change in the Curvature Parameter for Gains

\begin{tabular}{ccc}
\hline$\gamma_{G}$ & Welfare Loss (in \%) & CRRA Parameter \\
\hline 0.2 & 1.80 & 32.5 \\
0.3 & 2.26 & 21.3 \\
$\mathbf{0 . 4}$ & $\mathbf{2 . 9 6}$ & $\mathbf{1 4 . 5}$ \\
0.5 & 4.32 & 9.7 \\
0.6 & 7.88 & 6.3 \\
\hline
\end{tabular}

(d) Change in the Curvature Parameter for Losses

\begin{tabular}{ccc}
\hline$\gamma_{L}$ & Welfare Loss (in \%) & CRRA Parameter \\
\hline 0.75 & 6.48 & 3.5 \\
0.85 & 6.24 & 4.8 \\
0.95 & 5.60 & 6.5 \\
1.1 & 4.38 & 9.7 \\
1.2 & 3.62 & 12.0 \\
$\mathbf{1 . 3}$ & $\mathbf{2 . 9 6}$ & $\mathbf{1 4 . 5}$ \\
1.4 & 2.41 & 16.9 \\
1.5 & 1.95 & 19.3 \\
\hline
\end{tabular}

(e) Change in the Dynamics of the Reference Level

\begin{tabular}{ccc}
\hline$(\alpha, \beta)$ & Welfare Loss (in \%) & CRRA Parameter \\
\hline$(\mathbf{0 , 0})$ & $\mathbf{2 . 9 6}$ & $\mathbf{1 4 . 5}$ \\
$(0.05,0.04)$ & 10.70 & 6.9 \\
$(0.05,0.045)$ & 9.65 & 13.7 \\
$(0.1,0.07)$ & 19.37 & 3.7 \\
$(0.1,0.09)$ & 22.33 & 15.6 \\
$(0.2,0.19)$ & 18.76 & 26.3 \\
$(0.3,0.27)$ & 11.05 & 34.2 \\
\hline
\end{tabular}


Table 4:

\section{Sensitivity of Consumption Shocks to Current and Past Wealth Shocks}

The table reports the OLS coefficient estimates $\widehat{\beta}_{1}, \ldots, \widehat{\beta}_{5}$ of the following regression model:

$$
\Delta \log c_{t}^{*}=\sum_{i=1}^{5} \beta_{i} \Delta \log W_{t+1-i}^{*}+\epsilon_{t},
$$

where $\Delta \log c_{t}^{*}$ and $\Delta \log W_{t}^{*}$ denote the changes in $\log$ (demeaned) optimal consumption and $\log$ (demeaned) optimal total wealth between adult age $t-1$ and adult age $t$, respectively, and $\epsilon_{t}$ represents the error term at adult age $t$. We use simulated consumption and wealth data to estimate the regression model. The individual is currently aged 65 (i.e., $t=65-25=40$ ). The benchmark parameter values are as follows: $\left(\theta_{0}, \kappa, \gamma_{G}, \gamma_{L}, \alpha, \beta\right)=(\$ 36000,5,0.2,1.3,0.3,0.3)$. We set the parameter $L_{t}^{\max }-$ which denotes the maximum amount by which consumption can fall below the reference level - to $50 \%$ of the initial reference level. The last three columns show the impact of assuming a different $\alpha$ and $\beta$ on the OLS coefficient estimates. All the estimates are significant at the $1 \%$ level.

\begin{tabular}{cccccc}
\hline & & & \multicolumn{3}{c}{ Different $\alpha$ and $\beta$} \\
\cline { 4 - 5 } Variable & Benchmark & CRRA & $\alpha=\beta=0.05$ & $\alpha=\beta=0.15$ & $\alpha=\beta=0.6$ \\
\hline$\Delta \log W_{t}^{*}$ & 0.2006 & 1.0000 & 0.6577 & 0.3391 & 0.1141 \\
$\Delta \log W_{t-1}^{*}$ & 0.0531 & 0.0000 & 0.0177 & 0.0426 & 0.0626 \\
$\Delta \log W_{t-2}^{*}$ & 0.0497 & 0.0000 & 0.0176 & 0.0392 & 0.0583 \\
$\Delta \log W_{t-3}^{*}$ & 0.0463 & 0.0000 & 0.0168 & 0.0373 & 0.0539 \\
$\Delta \log W_{t-4}^{*}$ & 0.0442 & 0.0000 & 0.0166 & 0.0345 & 0.0501 \\
\hline
\end{tabular}

